\title{
15
}

\section{Hot Electron Nanoscopy and Spectroscopy (HENs)}

\author{
Andrea Giugni ${ }^{1}$, Bruno Torre ${ }^{1}$, Marco Allione ${ }^{1}$, Gerardo Perozziello ${ }^{2}$, \\ Patrizio Candeloro ${ }^{2}$, and Enzo Di Fabrizio ${ }^{1,2}$ \\ ${ }^{1}$ SMILEs Lab, Physical Science and Engineering Division (PSE), King Abdullah University of Science and \\ Technology (KAUST), Ibn Al-Haytham Bldg., Thuwal 23955-6900, Saudi Arabia \\ 2 Departement of Experimental Clinics, Bionem Lab, University Magna Graecia, Campus "Salvatore Venuta" \\ Viale Europa, 88100 Germaneto-Catanzaro, Italy
}

\subsection{Introduction}

The fields of plasmonics [1] and nanoelectronics [2] have attracted the interest of a wide audience over the past two decades triggered by the disclosure of many fundamental scientific breakthroughs. New ideas have led to fascinating experimental results in many fields in physics and biology, pushing to a new level the knowledge and control on the processes that rule optoelectronic devices and life science at the nanoscale $[3,4]$.

Scanning probe microscopy (SPM) has greatly improved since its first implementation [5], now offering several specialized techniques that can reveal important insights into the topographical, mechanical, electronic, and magnetic properties of materials at the micro- and nanoscale [6].

At the same time, Raman spectroscopy can be combined with SPM setup [7] reaching the sub-diffraction limit resolution and providing detailed molecular information, in some cases supplementing those obtained by other techniques, and in others adding original insights into the study of materials [8].

In the field of surface plasmon photonics, a plasmonic device is a tool that, taking advantage of enhanced interactions of light with metallic nanostructures, shows some new special abilities. In particular, due to their intrinsic electromechanical nature [9] the so-called surface plasmon polaritons (SPPs) can overcome both limits in the fundamental optical diffraction (Rayleigh criterion) and in the energy transport mean free path of the electrons in the metal. This provides an effective way to guide and concentrate energy at the nanoscale, where specific energy transfer processes take place. Thanks to field intensification [10] and nonlocal effects, efficient plasmonic substrates have been proposed for enhanced spectroscopies [11] and selective photodetection [12]. Beside the photonic generation channel, an SPP can decay in hot carriers $(h c)$, which can be used for transport measurements in parallel with optical characterization. Energy confinement and an effective hot charges generation rate 
have driven plasmonic-assisted chemical catalysis [13] and offered new tools in material characterization [14]. The strong coupling, polarization sensitivity, and nonlinearities [15] in plasmonic devices made possible applications in lasing media [16], beam shapers [11, 17], and ultrafast devices [18] as well as metamaterials [19].

In the past years, quantum mechanical nature of SPPs and medium was included in the theory, getting new insights into the description of the propagation and scattering processes of electrons and phonons. This allows, in a quantum mechanical framework, the description of the decay process into charge pairs, known as hot electrons (he $\left.{ }^{-}\right)$and hot holes, in a confined space [20] and in bulk media [21], where energy distribution, decay channels, relaxation times, and mean free paths can be retrieved by ab initio calculations [21, 22]. First principle quantum description allows accounting for material-specific features, like interband transitions in noble metals, which are precluded in simplified jellium model, thus providing insight into the dynamics of plasmonic $h c$ generation.

From an optical point of view, the property of localized plasmons and SPPs generates the near-field light, with wavelength well below the diffraction limit, with a local field enhancement $[23,24]$ that has opened the way to spectroscopic analysis of tiny amounts of matter, down to the single molecule detection limit [25].

In this context, we have recently proposed a multi-spectroscopy scanning probe apparatus [14] for the simultaneous investigation of chemical, structural, and charge transport properties at nanoscale, exploiting synergic phenomena. These are the low-loss propagation through adiabatic plasmonic compression of the SPPs that induces a field enhancement (energy focusing) [23, 26] with maximal momentum transfer at the interface, and the effect of quantum confinement on the $h e^{-}$generation rate (improved responsivity). All these terms allow the control of the extraction rate of hot charges in a nanometric confined space.

The functional element is a plasmonic device fabricated on an atomic force microscopy (AFM) probe, composed of a photon to SPP coupler (a grating or a photonic cavity) and a final conical tip where energy compression and charge generation occur. The injection direction, along the normal to the interface between the tip and the sample, provides the maximal photonic contribution to the $h c$ momentum exchange and increases the SSP conversion rate at the nanostructure apex. The energy confinement in a small volume $(<10 \mathrm{~nm}$ in depth) at the tip-sample interface maximizes the hot charges production rate and their injection probability before their thermalization in the metal. At the interface between the nanometric tip apex and the semiconductor, the momentum conservation rule relaxes, allowing the hot electron to access the required phase space for crossing the barrier. Further, the observed radiative scattering at the tip apex generates a sub-diffraction optical probe, which can be used with the hot electron current source for combined transport and optical spectroscopy. Such a configuration does not require any voltage bias for the injection of the charge through the Schottky barrier (SB) and benefits from the reduced depletion region (a few nanometers linear length and depth) that is jointly responsible for the high spatial resolution and current sensitivity.

The surface of the tip is made of gold, also ensuring good stability and biocompatibility. The customized tip is fully compatible with all AFM cantilevers and 
can be used in many of the operational modes of the instrument. In particular, the transport of hcs with high localization and collection efficiency through an SB was first accomplished in contact mode. An ultimate efficiency exceeding 30\% and a lateral resolution of $3 \mathrm{~nm}$ for a Schottky $\mathrm{Au}-\mathrm{GaAs}$ junction is reported. In the present contribution, we include our updated results on hot electron AFM nanoscopy.

We have organized the chapter to include a brief description of different laser coupling methods with guided SPP modes at the surface of a cone. We show some devices, their electromagnetic simulations, and their optical characterization. A theoretical section illustrates the optical and quantum description of the hot charge generation rate as obtained for the SPP propagation along the nanocone in adiabatic compression. We also show some experimental results concerning the application of the hot electron nanoscopy and spectroscopy (HENs) in the so-called Schottky configuration, highlighting the sensitivity and the nanoscale resolution of the technique. The comparison with Kelvin probe and other electric AFM techniques points out the intrinsic advantages of the HENs. In the end, some further insights are given about the possibility of exploiting HENs with a pulsed laser at the femtosecond time scale without significant pulse broadening and dispersion.

\subsection{Coupling Schemes}

According to their dispersion relations, there is a momentum mismatch between the photon and the SPP having the same energy, as shown in Figure 15.1a. Several coupling configurations have been proposed to couple the freely propagating photon with the SPP, fulfilling the energy and momentum conservation rules for the interaction process. The first constraint fixes the frequency, while the second determines the amount of momentum that must be provided, once the frequency-dependent SPP wave-vector dispersion is known [27]. An effective coupling is also responsible for an efficient hot electron generation.

As SPP launchers [28], we consider two methods: a diffraction grating consisting of a periodic modulation of the surface of the metallic device, based on the phenomenon named after W. Wood, and a photonic cavity of proper symmetry. Other configurations are possible, such as the use of a high-index glass prism, commonly used in the Kretschmann-Raether [29, 30] or the Otto [31] configurations.

The complete device consists of an AFM cantilever where a grating, acting as a coupler, is engraved by means of a focused ion milling process. The coupler is placed in the proximity of a micrometric metallic cone whose apex can be fabricated with a radius of curvature down to $5 \mathrm{~nm}$, as shown in Figure 15.3b. According to our scattering geometry, which fixes the incident angle $\theta_{\text {in }} \sim 36^{\circ}$ with respect to the normal of the grating surface, we calculated the grating period $P$ allowing to compensate the momentum mismatch (Figure 15.1a) from

$$
\frac{2 \pi}{P}=\Delta K_{z}=K_{z}^{\mathrm{SPP}}-K_{z}^{\mathrm{Ph}}=\frac{\omega}{c} \sqrt{\frac{\varepsilon_{d} \varepsilon_{m}}{\varepsilon_{d}+\varepsilon_{m}}}-\frac{2 \pi}{\lambda} \sin \theta_{\text {in }}
$$


(a)

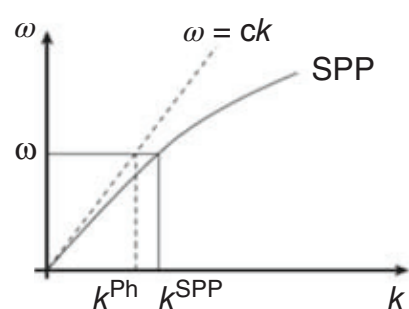

(b)

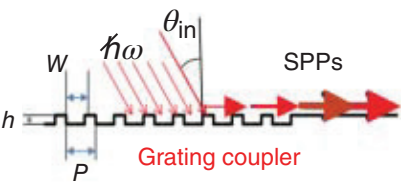

(c)

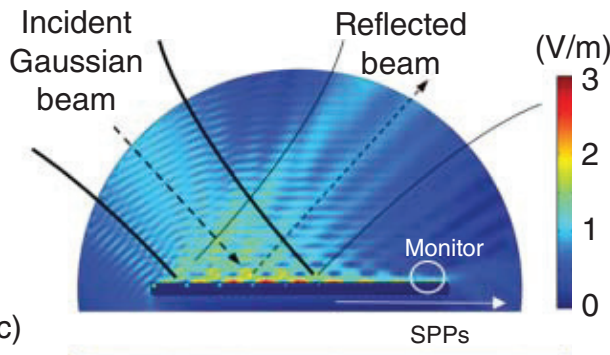

(d)

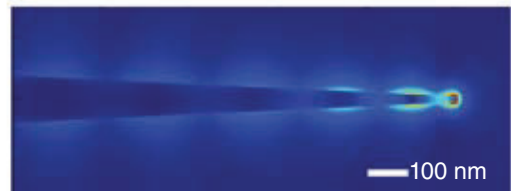

Figure 15.1 (a) The graph shows the momentum mismatch $K^{\mathrm{SPP}}-K^{\mathrm{Ph}}$ between the dispersion curve of an SPP mode and photon at a fixed angular frequency $\omega$. (b, c) Represent, respectively, the grating coupling geometry and the corresponding EM simulation. There are indicated the four meaningful parameters for the optimal coupling and the monitor position used for the energy flow analysis described in the text. The free parameters are the groove-pitch $P$, the height $h$, the width $w$, and the incidence angle $\theta_{\text {in }}$. (d) The E field amplitude in the propagation-induced adiabatic compression of a TMO mode along a plasmonic taper.

being $\lambda$ the free space wavelength, $K_{Z}^{\mathrm{SPP}}$ and $K_{Z}^{\mathrm{Ph}}$ the wavevector of the plasmon and of the photon along the $Z$ propagation direction, respectively, and $\varepsilon_{\mathrm{m}}$ and $\varepsilon_{\mathrm{d}}$ the frequency-dependent permittivity of gold and the dielectric material, respectively.

A 2D numerical simulation for six equally spaced grooves, performed using the COMSOL Multiphysics finite element method software, allowed us to optimize all the three significant parameters of the grating, that are, the period $P$, the height $h$, and the width $w$ of the grooves for the geometrical configuration $[34,35]$. The coupler, sketched in Figure 15.1b, was then realized on the flat sides of the tip, as shown in Figure 15.3b and c. Our simulations in Figure 15.1b show that about $10 \%$ of the incident radiation transfers to the propagating SPPs. We evaluated by means of Poynting vector analysis the efficiency of the coupling as the ratio between the energy flowing along the metal surface and the incident one, as indicated in Figure 15.1c. To estimate the propagation of SPPs to the tip apex, we performed a complete 3D electromagnetic simulation of the whole device, with the optimal parameters calculated for a wavelength of $670 \mathrm{~nm}$ $(P=1550 \mathrm{~nm}, h=150 \mathrm{~nm}, w=220 \mathrm{~nm})$. We considered a Gaussian beam with $E_{0}=1 \mathrm{~V} / \mathrm{m}$ on the axis, and the whole device was immersed in a dielectric medium of refractive index $n=1$. We inserted five layers of perfect matching layers (PMLs) around the simulation volume, far away from the metal structures to avoid spurious effects between the evanescent waves and the PML. The electromagnetic simulation allows estimating the amplitude of the electric field at the apex (tip curvature $\sim 12 \mathrm{~nm}$, as the experimental value of the tip used for the measurement reported in Section 15.5) of the conical structure extending about $2.5 \mu \mathrm{m}$ over the AFM pyramidal tip. The cone promotes the axial symmetric TM0 propagating mode, a transverse magnetic mode with a radially polarized electric field with respect to the cone axis [36]. The result, 
reported in Figure 15.1c as a zoomed image of the tip, reproduces the so-called adiabatic compression phenomenon, which describes the slowing down to zero of both group and phase velocity of the SPP proceeding along the cone toward the tip apex, with a temporal logarithmic dependence [23].

Another possible coupling configuration, favorable from an experimental point of view, relies on the use of a photonic crystal $(\mathrm{PhC})$ slab cavity coaxial with the cone and in contact with its base [37]. In this case, the cone can be fabricated directly on a tipless AFM cantilever. Optimal coupling for the TM0 mode along the cone surface can be obtained with the use of a so-called PhC-H1 structure, a PhC cavity with a hexagonal periodicity of holes in the dielectric and with a single missing hole that defines the optical cavity center. This $\mathrm{PhC}-\mathrm{H} 1$ selects a radial mode when illuminated with linear polarized light, for example, through a microscope objective, as shown in the drawing in Figure 15.3a.

In Figure $15.2 \mathrm{a}-\mathrm{e}$ we report the electromagnetic simulation of the cavity proposed in Ref. [37] when illuminated with $633 \mathrm{~nm}$, while in Figure 15.3a is the

(a)

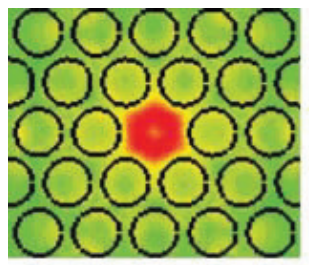

(d)

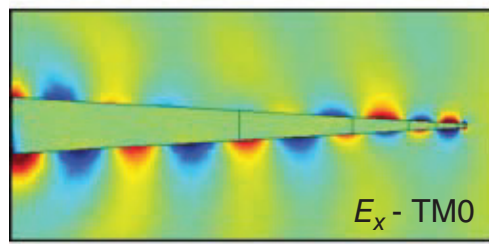

(f) 1.5

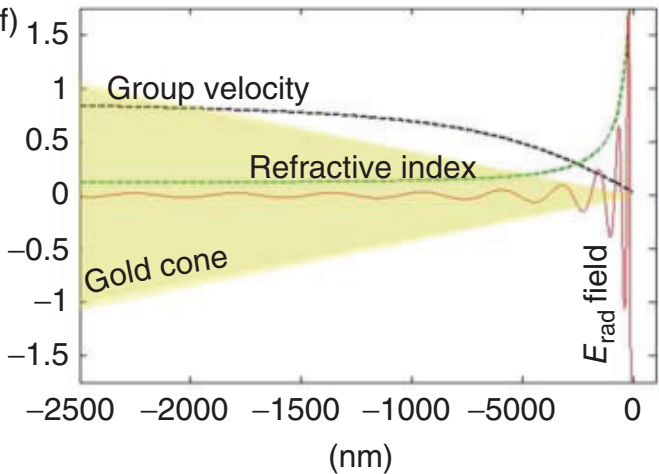

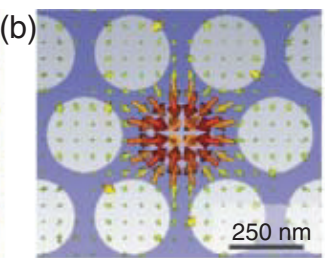

$[\mathrm{V} / \mathrm{m}]$

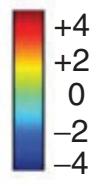

(g)
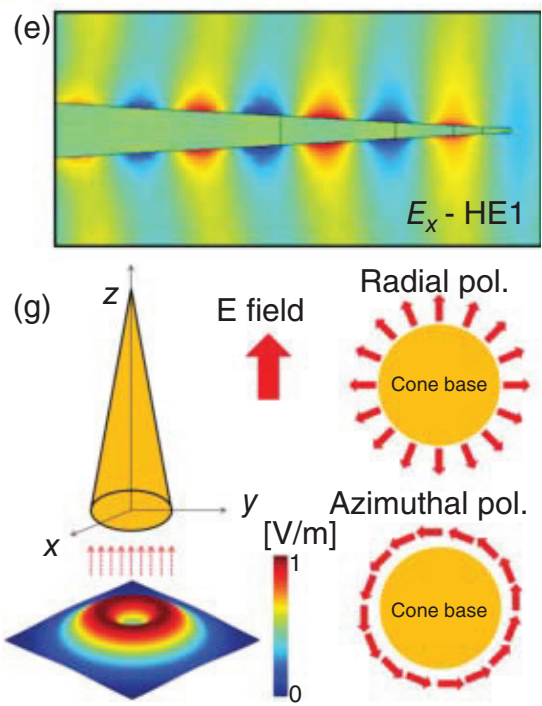

Figure 15.2 (a) Total electric field at $z=z_{0}$ (slab thickness) for the photonic crystal slab of Figure 15.3a supporting radial modes at $\lambda=633 \mathrm{~nm}$. (b) Vectorial plot of the electric field at $z_{0}$ evidencing the radial TMO structure of the mode. (c) Full SPP propagation and field calculation for a silver nanocone on $\mathrm{H} 1$ radial cavity $(\mathrm{d}, \mathrm{e})$ radial component of the electric fields of TM0 and HE1 modes propagating along the conical tip. Only for the TMO case is observable the focusing and the progressive reduction of the effective wavelength. (f) Simulated $E_{r}$ field for a TMO mode in adiabatic compression, there are graphed also the group velocity, with respect to light speed in vacuum, and the effective refractive index (10x). (g) Coupling scheme of a radial polarized mode at the cone base. (a-c) Reproduced with permission from [37], copyright OSA 2011. (d-g) Reproduced with permission from [33], copyright IOP 2014. 

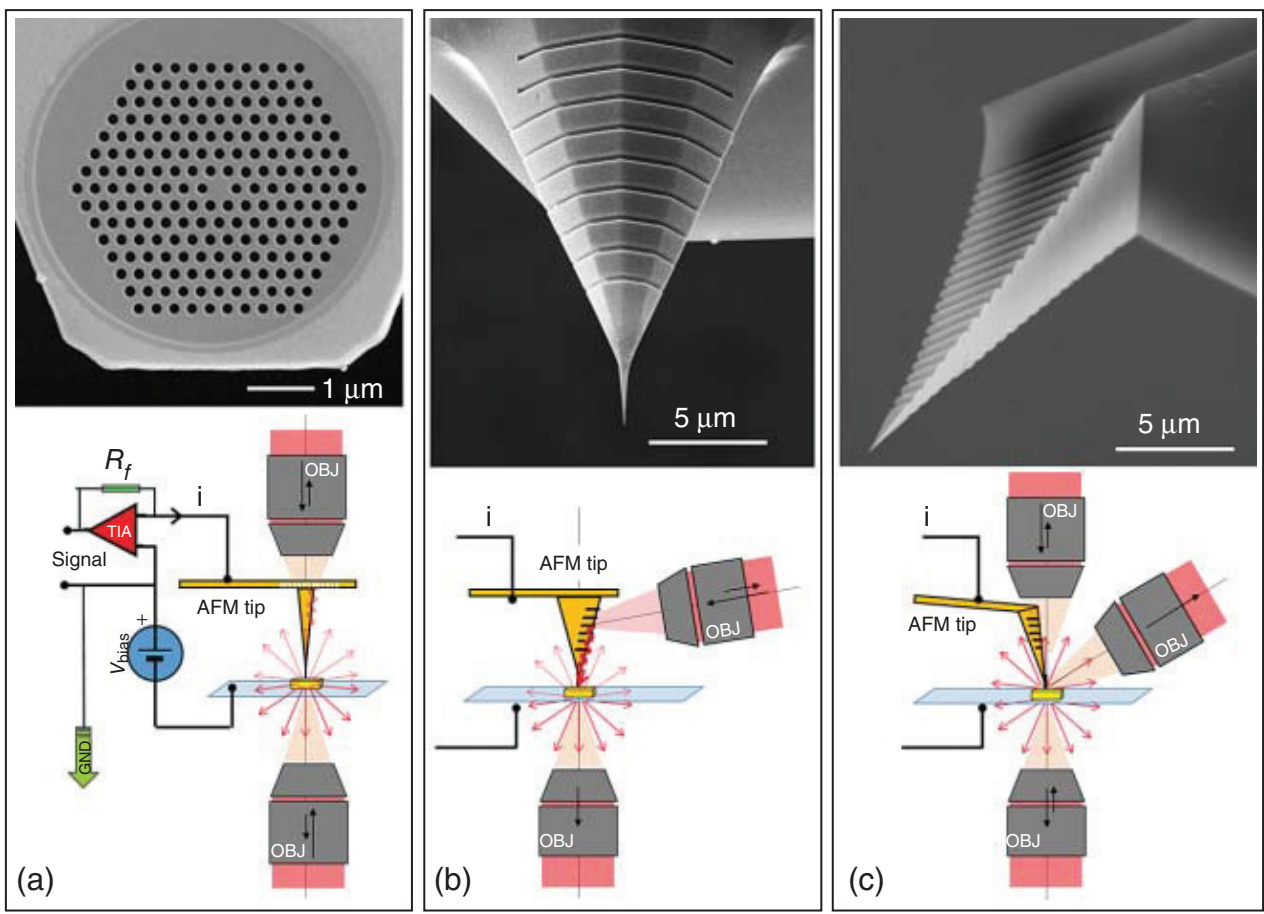

Figure 15.3 Devices promoting adiabatic compression and corresponding possible coupling geometries. (a) SEM micrograph of $\mathrm{PhC}-\mathrm{H} 1$ realized on a silicon AFM cantilever used to generate an axially symmetric mode. Using the illumination geometry indicated, the $\mathrm{H} 1$ photonic cavity allows to couple a nanocone with laser beam, squeezing the spot dimension below the diffraction limit. (b, c) SEM micrographs of devices for non-local excitation of the tip apex mediated by a grating coupler. Alternative configurations for maximal sample optical accessibility are displayed. In the pictures, the black arrow in correspondence of the objectives specifies if they are for excitation detection or both. From polarization considerations the configuration in (b) is to be preferred. (a) Reproduced with permission from [32], copyright NPG 2010. (c) Reproduced with permission from [33], copyright IOP 2014.

scanning electron microscopy (SEM) image of a $\mathrm{PhC}$ example. In this case, the material was silicon with a refractive index $n_{633 \mathrm{~nm}}=3.917+i 0.0122$. The periodicity was $P=290 \mathrm{~nm}$ with hole radius $R=0.4^{*} P$ and slab thickness $z_{0}=162 \mathrm{~nm}$. The finite difference time domain (FDTD) simulation (CST package) of the devices clarifies the symmetric structure of the sustained pure radial mode. In particular, the electric field distribution of a TM0 mode is shown in Figure 15.2a and its vectorial structure in Figure 15.2b. The simulated cone is made up of silver, $\varepsilon=-14.469+i 1.094$, and has a base diameter of about $300 \mathrm{~nm}$ and a height of $2.5 \mu \mathrm{m}$. For a $\mathrm{PhC}-\mathrm{H} 1$, the field has a pure TM0 structure, both at the base of the cone, where the SPP is launched, and at the apex. Figure $15.2 \mathrm{~d}$ and e shows the value $E_{z}$ of the electric field along the cone when either a radially polarized source or a plane wave impinges on the base of the cone. For both cases, the direction of propagation of the illumination is parallel to the axis of the metallic cone, and the laser spot size matches the cone base. As expected, the final field of the SPP and its mode strongly depend on the source symmetry. The plane wave generates an HE1 mode that does not show any compression. 

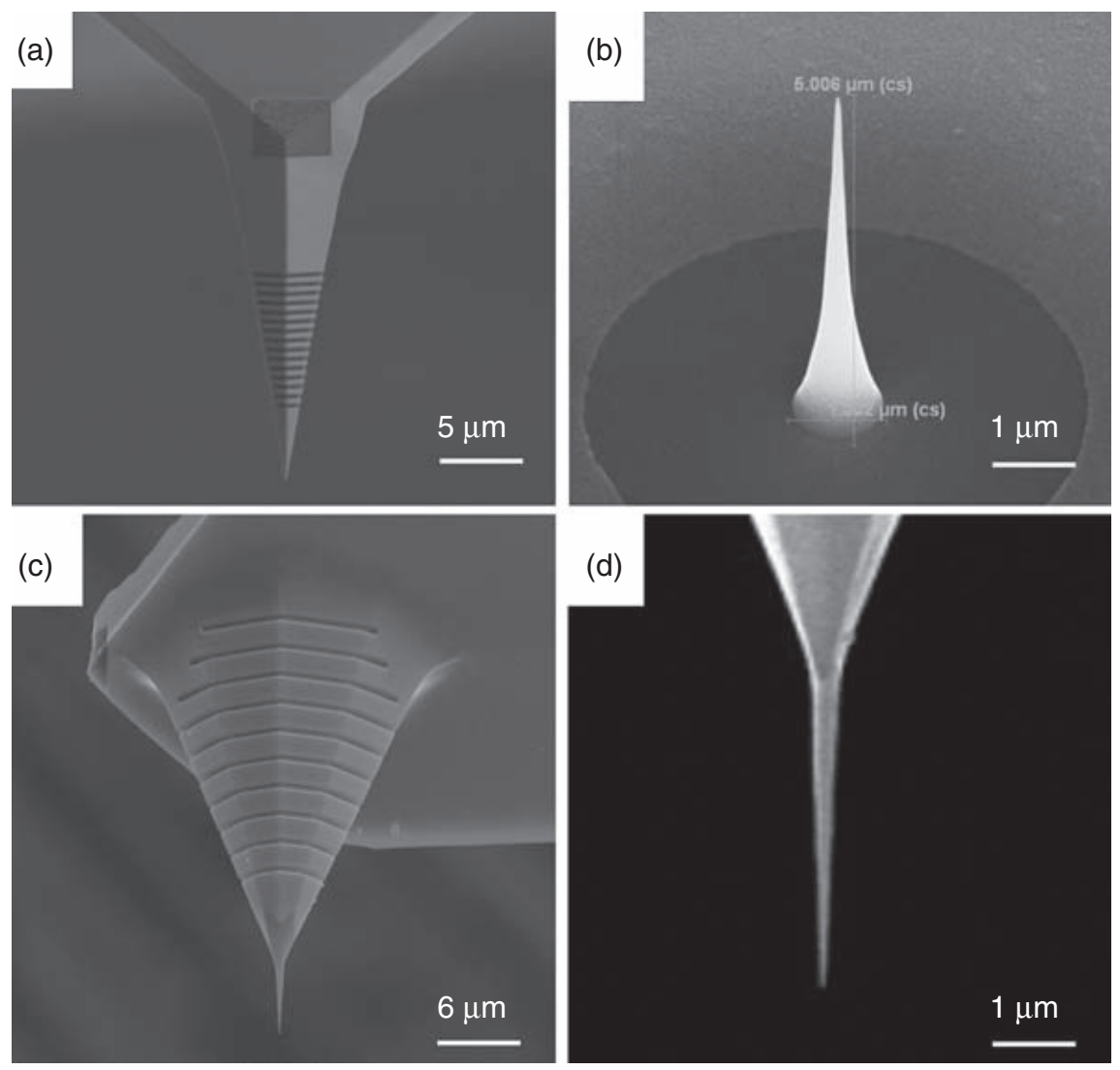

Figure 15.4 Scanning electron micrographs of three different devices used for non-local excitation of the tip apex. (a) SEM image illustrating the fabrication of the grating for back propagation of SPPs at the wavelength $670 \mathrm{~nm}$, as in Figure 15.3c. (b) SEM image of the sharp cone grown on a suspended thin $\mathrm{Si}_{3} \mathrm{~N}_{4}$ membrane. (c, d) One of the devices and the detailed image of its apex used for the hot electron imaging reported in this paper. The devices were prepared by focused $\mathrm{Ga}^{+}$ions beam milling and electron-induced deposition, preceded and followed by gold sputtering. (c, d) Reprinted with permission from [14], copyright NPG 2013.

Figure $15.2 \mathrm{f}$ shows the characteristic effective wavelength, the group velocity, the refractive index, and the radial electric field of an SPP that undergoes adiabatic compression in the material.

For both structures, after successful coupling, the SPP propagates with a negligible back reflection and dissipation with a progressive enhancement of the electric field and an increase of the wave vector while approaching the apex of the cone.

In the optical configuration shown in Figure 15.3a, it is also possible to directly illuminate the edge-base of the cone with a radial polarized beam. In this case, the cone can be fabricated on a tipless AFM cantilever, as shown in Figure 15.4b. The same edge relaxes the wavevector matching conditions, while the shape of the optical mode and its polarization symmetry match directly the cone geometry and the field symmetry constrains, as shown in its optical characterization in Figure 15.7. 


\subsection{Plasmonic Device and Optical Characterization}

When the size of our plasmonic devices approaches the bulk skin depth, the size that defines the geometry of the device becomes the only important length scale parameter. At this point, the SPP localization depends only on our ability to manufacture small objects. The theoretical picture at the base of this concept is detailed in Section 15.4.

The fabrication of the devices, reported in Figure 15.7, required a focused $\mathrm{Ga}^{+}$ ion milling (focused ion beam (FIB)) at $30 \mathrm{keV}$ to fabricate the coupling grating and the photonic cavity, followed by an intermediate gold/silver coating process and a subsequent electron-beam-induced deposition at $5 \mathrm{keV}$, to grow the cone using a Pt-C precursor gas. The final step consisted in a noble metal deposition of $\sim 50 \mathrm{~nm}$ on the device by sputtering, with a custom procedure that maintains the tip apex radius in the range of a few nanometers. These steps provide an effective coupling between tip and cone. The control of the groove width is $\pm 5 \mathrm{~nm}$, while the depth was realized with a reproducibility of $\pm 15 \mathrm{~nm}$. SEM images of some examples of the final devices obtained for different coupling geometries are reposted in Figure 15.3 and 15.4.

Important aspects to control are the symmetry of the apex, the homogeneity of the coating, the final radius of curvature, and the lack of asperities, which act as unwanted scattering centers in the proximity of the tip apex. Moreover, a smooth connection between the grating launcher, usually fabricated on a side of the commercial tip, and the base of the cone has to be obtained to minimize the impedance mismatch along the SPP propagation direction. Finally, the tip shape has to be preserved during scanning; therefore, the cone hardness and the metal coverage at the apex, which are the elements that define the Schottky contact [38], have to be realized with high uniformity and control over material growth. For the same reason, the use of very low spring constant cantilevers, about $0.1 \mathrm{~N} / \mathrm{m}$, is suggested, since lower loads are more easily achievable while working in contact mode.

Once these parameters have been optimized, it is possible to reach a final efficiency very close to the theoretical limit, around $30 \%$, and $h e^{-}$responsivity in the order of $\mathrm{nA} / \mu \mathrm{W}$, with a very good scanning stability, comparable to the one obtained with commercial probes used in other electrical mode configurations.

An optical characterization follows the fabrication phase. To routinely check the optical performances, we have built up an ad hoc setup constituted by three optical microscopes along $X, Y, Z$ orthogonal axes, all imaging the same spatial volume where a tip can be positioned (Figure 15.5f). The tip stage has six degrees of freedom $(X, Y, Z, \theta, \phi, \alpha)$ and the laser illumination, with tunable polarization, is aligned coaxially with one of the optical paths. Each microscope has a calcite displacer to image the two polarizations of the scattered light at the same time. In general, a calcite displacer should be avoided in white-light imaging, since it acts as a dispersive prism for the displaced beam. Nevertheless, the use of monochromatic excitation maintains the same focal plane, resulting in a parallel acquisition of the two different polarizations for the image, and thus providing a good solution for the polarization analysis of the light scattered from 

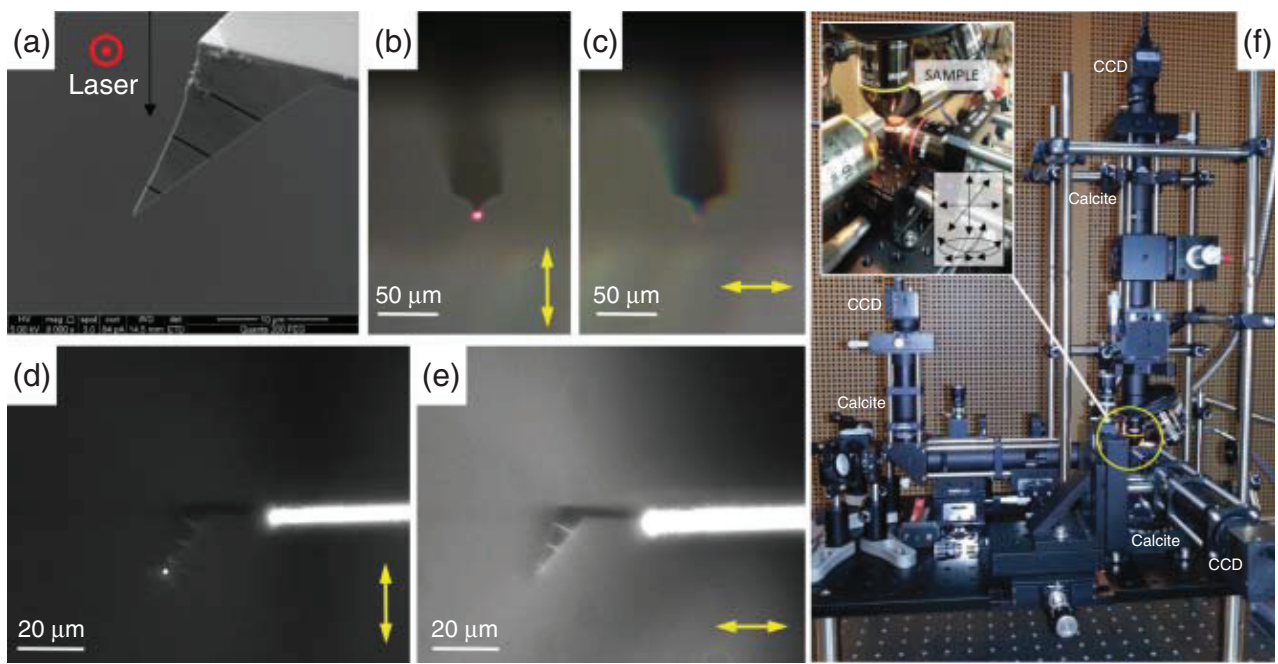

Figure 15.5 (a) SEM image of a grating coupler realized on a nose-type tip for top illumination. Direction and polarization of exciting laser is also shown. (b-e) Refers to the test of the grating coupling on nose-type tip (Nanosensors ATEC-contAu cantilever) for $\lambda=633 \mathrm{~nm}$ top side illumination. $(b, c)$ Optical images of the tip as seen from the front and analyzed for two hortogonal polarizations as indicated by yellow arrows. $(d, e)$ Side views of the tip. A background far field white light illuminates the overall tip and cantilever profiles. (f) Photograph of the optical setup; inset shows the sample area.

the device. In Figures 15.5 and 15.6, we report the SEM micrographs and optical characterization of two devices similar to the ones used for the experiment reported below.

Figure 15.7 illustrates the excitation of the axially symmetric SPP mode with 647-nm laser on a silver cone realized on suspended $\mathrm{Si}_{3} \mathrm{~N}_{4}$ membranes. In this case, a specific optical setup for the control of the polarization of the incident beam and the nanopositioning of the cone has been realized; it is illustrated schematically in Figure 15.7d. The large apertures on both sides of the metal act as diaphragms for the focused impinging beam, allowing to locally optimize the radial mode profile matching the cone base edge (Figure 15.7c). By changing the polarization of the incident beam, it is possible to move continuously from pure radial to pure azimuthal polarization, while monitoring at the same time the SPP propagation along the cone by imaging the tip plane, as shown in Figure $15.7 \mathrm{f}-\mathrm{h}$.

\subsection{Theoretical Section}

The absorption of a photon by a metal surface, as described, can generate an SPP [39]. An SPP is defined as a nonequilibrium collective electron gas excitation in the metal, oscillating at the electromagnetic frequency, that propagates confined along the metal-dielectric interface for tens of micrometers before some scattering event damps it, allowing for its decay into energized charges or a new photon. Due to the nature of the coupling, the optically generated SPP has intrinsically low momentum resulting in a nonfavorable condition for the SPP $h e^{-}$scattering. This gives to SPPs an exceptionally long mean free propagation length, compared to the one of a single electron. 

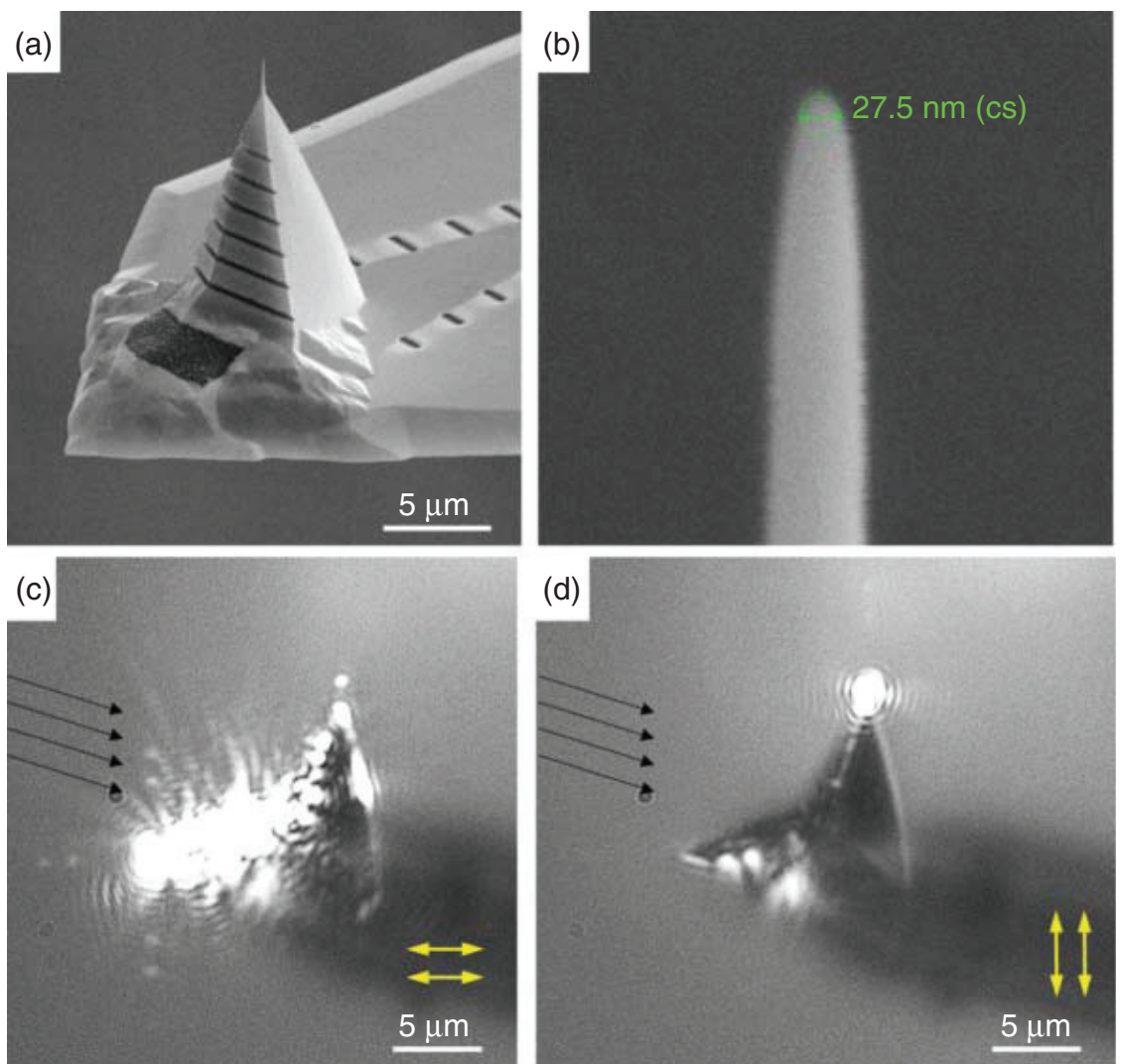

(d)

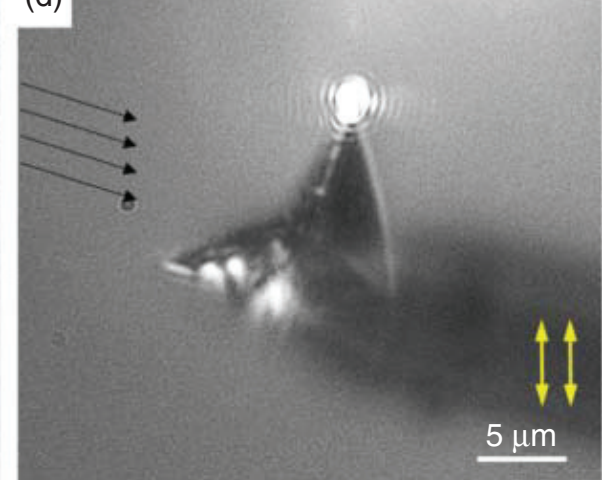

Figure 15.6 (a) Full device structure fabricated considering optimal value of the simulated parameters for a wavelength of $633 \mathrm{~nm}$ and illumination direction as depicted in (c, d). (b) Detail of the apex of the cone of (a). (c, d) Optical characterization showing, respectively, the horizontal or the vertical polarization in excitation and in analysis at the wavelength of $633 \mathrm{~nm}$, as indicated by the arrows. Different scattering intensities reflect the prevailing mechanism for the two polarizations: scattering in (c) and coupling in (d). A background far-field white-light illumination allowed capturing the overall tip profile. The vertical excitation, correctly, induces a strong scattering from the apex, confirming the SPP propagation described in the text. The device shown here was realized on Micromash CSC38 cantilever. The cone apex has an estimated radius of curvature $<15 \mathrm{~nm}$.

Coupling, propagation, and damping of an SPP are ruled by complex dynamics, strongly depending on the intimate nature of the medium. The intrinsic nature of SPPs physically bridges different length scales, from the micrometric to nanometric: by nanostructuring the specimen at those length scales, it is therefore possible to show different aspects of this complexity to underline the huge richness of phenomena and valuable experimental potential for different applications. The dynamic processes that regulate $h c$ generation in noble metals, their propagation, and possibly their extraction are attracting an increasing interest in both the theoretical research and applied fields. Furthermore, another important emerging research technique is the realization of a multi-spectroscopies probe, with improved efficiency for the investigation of the matter at the nanoscale.

Despite very significant experimental and theoretical efforts, a comprehensive description of the processes that determine the measure of a net photocurrent is still not clearly given at the tip of the adiabatic nanocone. Here we recall classical 

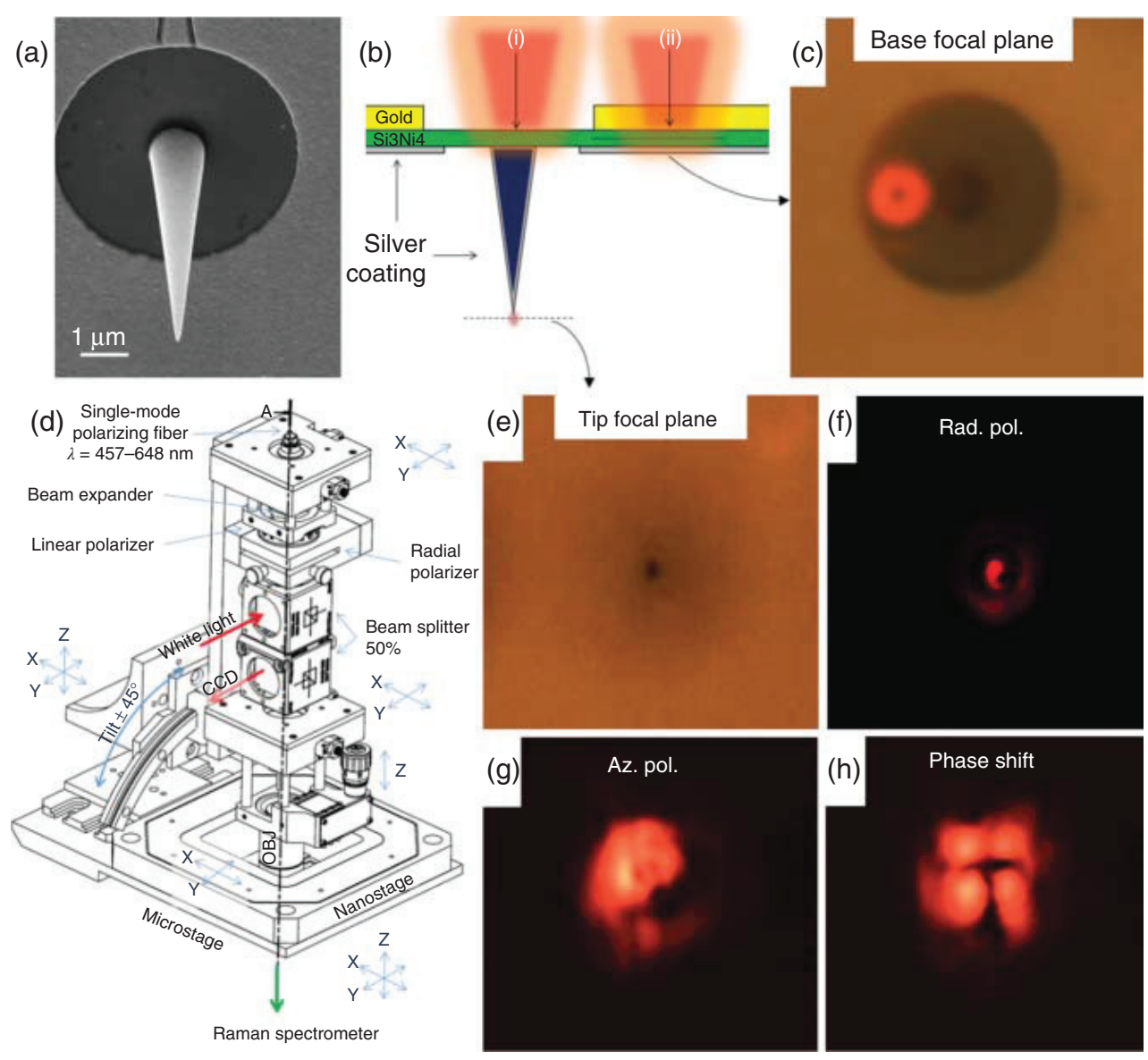

Figure 15.7 (a) SEM micrograph of a silver cone, fabricated by FIB and sputtering techniques, on $\mathrm{Si}_{3} \mathrm{~N}_{4}$ membrane (500-nm thick) and supported by Si wafer promoting adiabatic compression. (b) Corresponding coupling geometry. Direct coupling at the base requires the dimension matching between spot and cone base diameters. In (d), we report the mechanical design of the optical setup used to excite SPPs along the cone by means of direct illumination with a radial polarized optical mode. The main optical elements are indicated in the picture. The system allows converting continuously a linear polarization into a radial or azimuthal one. A fine nano-position of the sample allows checking the robustness of this type of coupling. Two nonpolarizing beam splitter cubes accommodate the optical path for a charge-coupled device (CCD) imaging system and a 50x objective allows focusing light on the back of the cone. A second objective collects the images from the bottom, facing the tip of the cone, as shown in (c, e-h). (c, e) The focal planes, collected in white light, at the base and top of the cone. (c) The characteristic donut intensity profile of the circularly polarized spot at $647 \mathrm{~nm}$ in its focal plane. ( $f-h)$ The scattered light pattern as can be collected from the tip focal plane. When radial polarization is used, the coupled SPPs propagate adiabatically to the apex originating a point source useful for spectroscopic applications.

and quantum mechanical descriptions that are relevant to the explanation of the phenomena $[20,21,23,40-50]$.

\subsubsection{Semiclassical Considerations}

A classical electromagnetic model (EM) assumes the continuity of the medium and describes a noble metal in a jellium framework as a free-electron-like system, 
with a parabolic dispersion curve for the conduction electrons. The adoption of experimental complex frequency-dependent dielectric function allows for the description of the interaction between the electromagnetic field and the metallic guide, introducing geometrical factors.

The EM description of a tapered plasmonic guide, terminating with apex dimension of a few nanometers, has led to the so-called adiabatic nanocompression idea, introduced for the first time in a pioneering work by Stockman [23] in 2004. Such a transport mechanism determines the concentration of the SPP energy at the tip, with a corresponding local field enhancement by about three orders of magnitude. The spatial confinement breaks the diffraction limit and opens up the experimental capability to harvest energy along the nanostructure, in the form of SPPs, and to bring it up to the very tip apex with negligible losses. All these phenomena cooperate to realize a sensor, which is capable of probing materials at the apex by means of both electromagnetic field and electronic transport.

We can assume, in general, that the creation of $N$ electron-hole pairs along the taper, resulting from the damping of SPPs can be described by means of its rate [23]

$$
\dot{N}=\mathrm{d} N / \mathrm{d} t=2 k^{\prime \prime} \frac{P}{\hbar \omega} \Delta z
$$

where $\omega$ is the angular frequency, $k^{\prime \prime}$ is the imaginary part of the complex wave vector $k$, and $P$ is the optical power of the SPP. $\Delta \mathrm{z}$ is the distance of the electron mean free path, $\sim 20 \mathrm{~nm}$ in our case (gold in the near-infrared range (NIR)). For a taper with a nanoscale radius $k_{0} R \ll 1, k_{0}$ being the plasmon wavevector on a flat surface, the SPP dispersion relations are well described by the analytically approximated formula

$$
k=k_{0} n(R) \approx \frac{1}{R}\left[-\frac{\varepsilon_{m}}{2 \varepsilon_{d}}\left(\ln \sqrt{-\frac{4 \varepsilon_{m}}{\varepsilon_{d}}}-\gamma\right)\right]^{-1 / 2}
$$

where $R$ is the radius of curvature of the tip, $\varepsilon_{m}$ and $\varepsilon_{d}$ are the metal and air dielectric functions, respectively, and $\gamma$ is the Euler constant. It is then possible to predict that the generation rate increases as $R^{-1}$ in the last few tens of nanometers close to the tip apex.

Equivalently, we can evaluate the SPP attenuation length in a metallic conical structure at the tip. The skin depth, $l_{\text {skin }}$, that reflects the evanescent nature of the SPP at the interface in the perpendicular direction to the metal surface, is commonly expressed for a Drude metal by the formula

$$
l_{\text {skin }}^{i}=\frac{\lambda_{0}}{2 \pi}\left(\frac{-\varepsilon_{i}^{2}}{\varepsilon_{m}+\varepsilon_{d}}\right)^{-1 / 2}
$$

where the index $i=m$ and $d$ accounts for the metal or the dielectric material.

Considering that $\left|\varepsilon_{m}\right| \gg 1$, this expression leads to a great concentration of the SPP near the interface. Recalling that $\varepsilon=n^{2}$ for $\mu=1$, neglecting absorption and using the expression of $k$, the penetration depth at the tip apex can 
be expressed as $l_{\text {skin }}^{m} \sim \frac{\lambda_{0}}{2 \pi}\left(\frac{1}{-\varepsilon_{m}}\right)^{1 / 2}=\frac{\lambda_{0}}{2 \pi n(R)} \cong R$, which states that an additional strong confinement takes place, reducing the penetration of the SPP to only a few nanometers below the surface.

Within a bulk picture for the electron scattering, we assume that each electron of the metal can be excited from any level of the conduction band with the same probability and that the $h e^{-}$are generated isotropically. Under this hypothesis, the probability for an $h e^{-}$to be transferred from the metal into the semiconductor is

$$
\eta=\frac{1}{2 n} \int_{0}^{\pi / 2} d \theta \sin \theta \int_{0}^{\varepsilon_{F}} d \varepsilon \rho(\varepsilon) \Theta\left((\varepsilon+\hbar \omega) \cos ^{2} \theta-\left(\varepsilon_{F}+e \phi_{b}\right)\right),
$$

where $\theta$ is the angle to the normal to the junction through which the electron is emitted (Figure 15.8d reports a schematic represetation of how such angle is determined), $\varepsilon$ is the initial energy of the electron, $\rho(\varepsilon)$, and $\varepsilon_{F}$ are the density of states and the Fermi energy of the metallic coating of the tip, respectively (i.e., SPP taper), $e \phi_{b}$ the height of the SB (see Figure 15.8b), and $\Theta$ is the Heaviside unit-step function. The factor one-half takes into account that only half of all the hot electrons propagate through the junction, while the second half does not contribute to the Schottky current as they propagate in the wrong direction. This equation states that only a part $(\varepsilon+\hbar \omega) \cos ^{2} \theta$ of the energy of electrons (the motion in the direction of the junction) is to be compared with the height of the SB.

Taking into account that in the NIR range $e \phi_{b}, \hbar \omega \ll \varepsilon_{F}$, some useful approximations can be done, the density of states can be taken at the Fermi surface, that is, we can set $\rho(\varepsilon) \approx \rho\left(\varepsilon_{F}\right)$, and we can write down for the electron energy $\varepsilon$ and the incidence angle $\theta$ the inequality $\cos \theta \geq 1-\frac{\hbar \omega-e \phi_{b}}{2 \varepsilon_{F}}$. In this limit, the probability distribution can be integrated to obtain the efficiency $\eta$ for the electron transport through the Schottky junction as

$$
\eta=\frac{\left(\hbar \omega-e \phi_{b}\right)^{2}}{4 \varepsilon_{F} \hbar \omega} \cdot \Theta\left(\hbar \omega-e \phi_{b}\right)
$$

which has the same quadratic dependence on electron energy as the well-known Fowler formula [51]. The previous result is obtained under the assumption of the conservation of the electron momentum in the plane of the junction. If such conservation is relaxed at the junction by roughness and curvature at the very tip of the nanostructure, then the probability of the electron transfer is significantly increased. In this case, we have

$$
\eta=\frac{1}{2 n} \int_{0}^{\pi / 2} d \theta \sin \theta \int_{0}^{\varepsilon_{F}} d \varepsilon \rho(\varepsilon) \Theta\left(\varepsilon+\hbar \omega-\left(\varepsilon_{F}+e \phi_{b}\right)\right)
$$

The integration now is trivial, and we obtain

$$
\eta=\frac{\hbar \omega-e \phi_{b}}{2 \hbar \omega} \cdot \Theta\left(\hbar \omega-e \phi_{b}\right)
$$

which is much greater with respect to the one of Eq. (15.6), since $2 \varepsilon_{F} \gg \hbar \omega-e \phi_{b}$.

Finally, responsivity $\rho$ and the total current $J$ across the Schottky junction can be related as

$$
J=e \eta \dot{N}=\rho P
$$


(a)

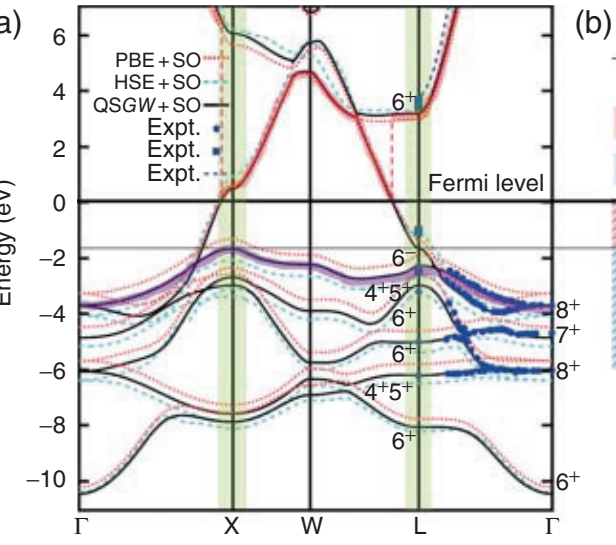

(c)

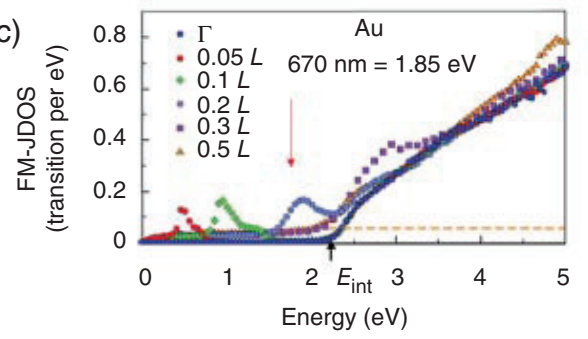

(b)

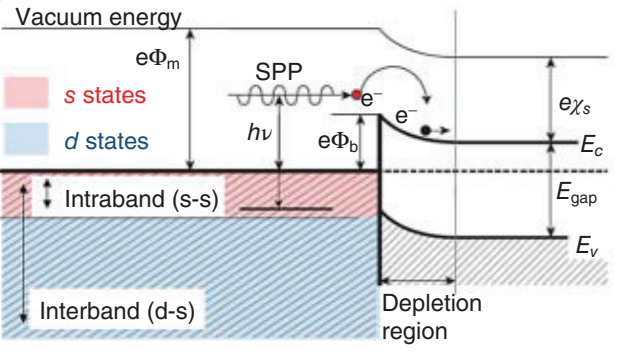

(d)

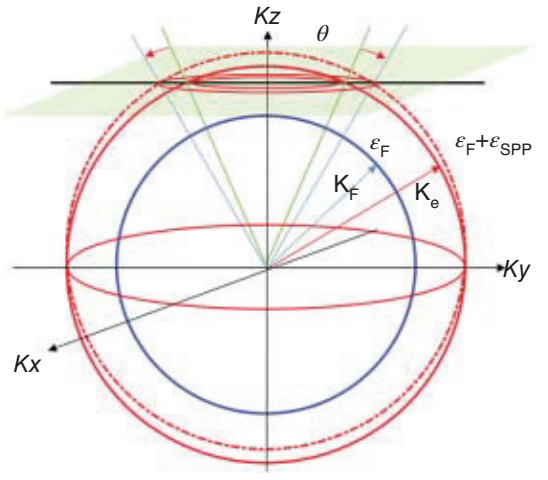

Figure 15.8 (a) Band structure of gold calculated within different many-body perturbation theory approximation. The zero of energy has been set at the Fermi level. The QSGW-SO, solid black lines, theoretical result best fit various experimental data from literature. Purple line specifies $5 \mathrm{~d}$ topmost occupied band, while red lines indicate $6 \mathrm{sp}$-like lowermost empty bands. Green vertical bands, about \pm one-tenth of $B Z$, representing the possible wavevector contribution provided by adiabatic compression. (b) Band scheme at metal/n-type semiconductor interface. We show the distribution of the conduction and valence bands in the semiconductor and energies of $\mathrm{s}$ and $\mathrm{d}$ states of gold. We also indicate the $h e^{-}$generation process with respect to Fermi level. (c) Finite momentum-joint density of states (FM-JDOS evaluated for different accessible electron wavevectors as a function of the energy. At about $2.1 \mathrm{eV}$, the interband energy indicates the onset of a characteristic linear dependence with the energy, typical of free electrons. In particular, a momentum of about 1/10 BZ allows for the efficient generation of energetic electron with energy around $1 \mathrm{eV}$ (green data). (d) Fermi sphere for the free electron model (blue). Schottky barrier (green plane) and the maximal electron energy after the SPP absorption (solid red circle). A rigid 1/10 BZ shift in the direction of the interface is also displayed (dotted red line). Panel a adapted with permission from Ref. [50], copyrighted by the American Physical Society. Panel c is reproduced with permission from Ref. [21], copyright American Institute of Physics 2015.

From these, we calculated the electron injection efficiency $\eta$ and responsivity $\rho$ at the tip by considering the two distinct experimental regimes described in the momentum exchange relations (15.6) and (15.7). As in the experiments, we considered a sample of $\mathrm{n}-\mathrm{GaAs}\left(N_{d}=3.6^{*} 10^{16} \mathrm{~cm}^{-3}\right)$, the Fermi energy level $\varepsilon_{F}=5.53 \mathrm{eV}$ of gold, and the dielectric function from Ref. [52], resulting in an $\mathrm{SB} e \Phi_{b}=0.865 \mathrm{eV}$. The radius of curvature of the tip and the electron mean free path were set to a conservative value of $R=20 \mathrm{~nm}$ and $\Delta z=25 \mathrm{~nm}$, the SPP optical power to $1 \mu \mathrm{W}$, deriving from a $10 \%$ of the laser power. The calculated transfer efficiency and responsivity are shown in Figure 15.12 as functions of wavelength $\lambda_{0}$, proving the quantitative agreement with the experimental values. Dotted and continuous blue lines in Figure 15.12a and b are pure $h e^{-}$contributions to the 
current when either the relation (15.6) or (15.7) is considered in the calculations, while the continuous red line in Figure $15.12 \mathrm{~b}$ accounts also for the photovoltaic contribution. We estimated the last term in the so-called point contact geometry limit that, departing from the planar model, describes the nanojunction in spherical coordinates and retrieves a depletion region extension as a spheroid with a remarkably shorter radius than the extension of the classical depletion region commonly used for an extended contact.

\subsubsection{Quantum Mechanical Considerations}

Experimental [53] and theoretical [21, 22] results show that the decay of SPPs with energy lower than the interband threshold in gold leads to the generation of long-living hcs with long isotropic mean free paths of up to $40 \mathrm{~nm}$ and energy within the $1-2 \mathrm{eV}$ range above $\varepsilon_{F}$. Moreover, promoting an SPP $(<2 \mathrm{eV})$ of wave vector of about $1 / 10$ of the Brillouin zone (BZ), as could be obtained from the expression of $k$ reported above, we could expect a maximum in the efficiency for $h c$ generation with an energy above $1 \mathrm{eV}$, that are well suited for $h e^{-}$scanning probe instrumentation, as can be deduced from Figure 15.8c.

Commonly, optically generated SPPs have wave vectors of 1/1000 of BZ. For them a decay process in hcs is highly unfavorable. Adiabatic propagation of a TM0 mode along a conical structure can easily increase plasmon momentum by a large factor while approaching the apex. This compression mechanism strongly depends on the last few tens of nanometers from the apex of the cone. For this reason, a proper fabrication procedure is crucial to obtain a sharp tip shape, as suggested by the theoretical model.

An important aspect in the adiabatic compression process is that the SPP skin depth reduces proportionally with the cone radius, meaning that at the apex a strong confinement is expected at the surface, with a behavior quite different from that observed for plasmonic thin films. The bulk SPP skin depth (13 $\mathrm{nm}$ at $\lambda=670 \mathrm{~nm}$ for gold) can rescale several times down to a few nanometers reaching the apex.

Even if commonly called free electrons, highly energetic carriers in noble metals are not completely free, but Bloch waves and their energy and momentum must fulfill the dispersion relation specific to the particular material and its band structure. As has been proposed for gold, phonon scattering contributes primarily to SPP decay in low-energy $h e^{-}(<2 \mathrm{eV})$ [21], differently from the common use of the Auger model. A similar behavior has been proposed for other noble metals.

The study of the band structure of gold, calculated in Ref. [50] using ab initio many-body perturbation theory (MBPT) compares several possible schemes in the approximation of the GW framework (G stands for the single particle Green's function and $\mathrm{W}$ for the dynamically screened Coulomb potential) including all relativistic effects, at least for the single particle. The quasiparticle self-consistent GW (QSGW) scheme, when taking into account all single-particle relativistic effects, particularly the spin-orbit (SO) coupling introduced on top of the scalar-relativistic approach, provides a theoretical band structure that better fits the experimental findings. This MBPT model results in optimal agreement with

experimental data from different techniques like the angle-resolved ultraviolet 
photoelectron spectroscopy shown in Figure 15.8a [50]. This QSGW-SO model lowers the $5 \mathrm{~d}$ bands, and globally achieves a considerable opening of the $5 \mathrm{~d}-6 \mathrm{sp}$ interband gap up to $0.8 \mathrm{eV}$, minimizing the discrepancy with the experiment. The QSGW-SO results are reported in Figure 15.8a along with experimental values. For our purpose, this work quantifies the band structure of gold and supports the interpretation of $a b$ initio MBPT calculation on SPP decay and energy spectrum of the generated hcs, as previously discussed.

\subsubsection{Quantum Confinement}

Here we consider the effects of quantum confinement on the energy distribution of $h c s$ [20] and on the injection across an SB. This contribution is considered fundamental in the description of experimental data for isolated nanoparticles and small clusters. For example, it can explain the enhanced photovoltaic effects, the Plasmon-induced $\mathrm{H}_{2}$ dissociation by catalytic effect of hcs [13], the size dependence of fluorescence and energy spectrum of hcs in quantum dots [54], and nanorods $[55,56]$. For this reason, we do not exclude its effect on the metal at the nanosized apex of the device. It is plausible that SPP spatial confinement also relaxes the wave vector constraint under a quantum mechanical point of view, that is, the wave vector of the hot electron particle can access a larger phase space. This fact contributes to match with the momentum requirement of $1 / 10 \mathrm{BZ}$, that is possible for about a spatial confinement of $\Delta x \sim 3 \mathrm{~nm}$. In this case, the occupation probability will change in favor of high-energy electrons.

The work of Govorov et al. [20] shows how an efficient generation of hcs can be obtained in small nanocrystals $(\leq 20 \mathrm{~nm})$ due to quantum confinement. In

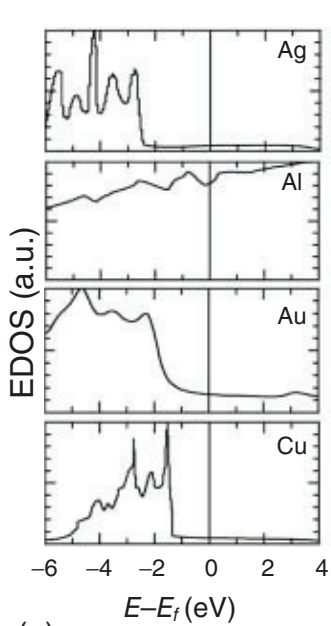

(a) (b)

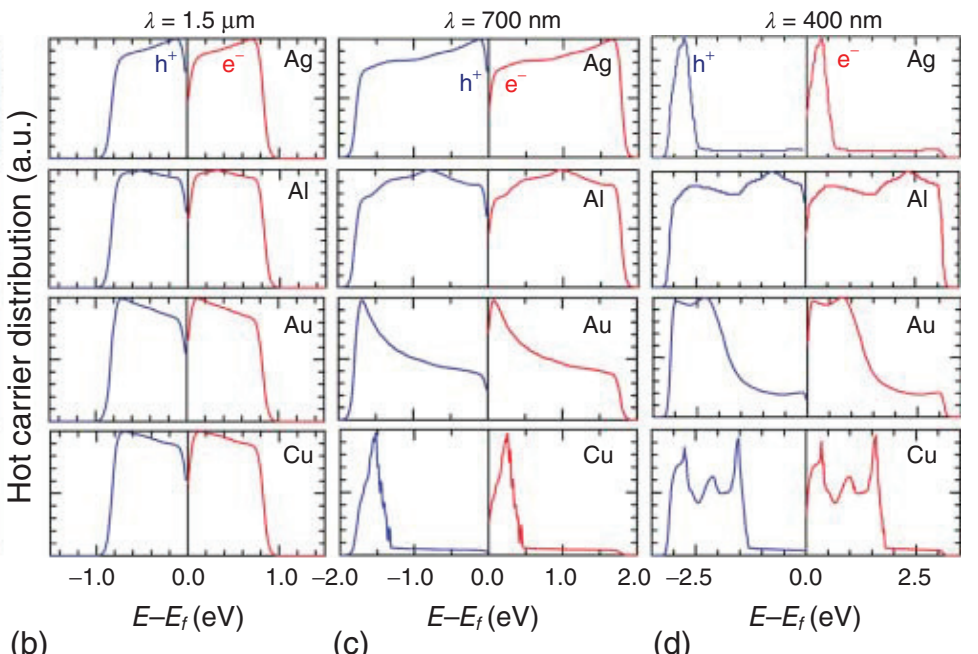

(c) (d)

Figure 15.9 Electron density of states (EDOS) and hot carrier distributions for common plasmonic materials: Ag, Al, Au, and Cu. (a) EDOS. Except Al (free electron-like), all exhibit a much higher density of states below the Fermi level. Under monochromatic illumination, HC distributions are created from incident photons with wavelengths: (b) $1.5 \mu \mathrm{m}(0.83 \mathrm{eV})$, (c) $700 \mathrm{~nm}(1.78 \mathrm{eV})$, and (d) $400 \mathrm{~nm}$ (3.11 eV). Low photon energies yield relatively uniform HC distributions. Upon higher energy, illumination peaks begin to appear due to high densities of occupied states below the Fermi level for Ag, Au, and Cu. Under $700 \mathrm{~nm}$ illumination, Au and $\mathrm{Cu}$ are more efficient for hole extraction than electron, being the hot hole distribution peaked farther from the Fermi level. Reproduced with permission from [57]. Copyright OSA 2015. 
this case, energy and momentum distributions for hcs shift toward higher values, largely improving the extraction yield. This work shows that photoexcited large nanocrystals produce a large number of low-energy hot electrons (few meV) and only a relatively small number of highly energized charges $(>1 \mathrm{eV})$, resulting in a not favorable condition for current extraction. Differently, an efficient generation and the subsequent injection of high-energy hcs can be realized exciting small nanocrystals, in particular when the optically induced electric current is along the smallest dimensions and it is normal to the collecting barrier interface.

These results are in agreement with those obtained for the bulk from ab initio calculations [21, 22], that is, also in the case of localized SPPs in nanocrystals, intraband transitions appear to be preferable for the generation of $h e^{-}$dominating the absorption for $\lambda>600 \mathrm{~nm}$, while interband transitions preferably generate energetic holes. This implies significant differences in the carrier generation and absorption spectra, as shown in Figure 15.9.

All these contributions together increase the final quantum efficiency of the device with respect to a bulk or to a classical nanowire.

\subsection{HENs Measurements: Plasmon-Assisted Current Maps and Ultimate Spatial Resolution}

Details about the experimental setup and the current to voltage I-V characterization of an $\mathrm{Au} / \mathrm{n}$-type GaAs nanojunction can be found in Refs [14, 33]. Here, we briefly recall the experimental setup architecture: we modulate the amplitude of the laser light while scanning in contact mode, as shown in Figures 15.10 and 15.13, measuring simultaneously the tip-sample current. Therefore, it is possible to discriminate and acquire at the same time the classical conduction and the $h e^{-}$map, for any arbitrary polarization of the junction, being the $h c$ signal the only one synchronous with the laser. Here, the hcs generated by plasmon damping enhance the internal photoemission (IPE) [58], a process that improves largely the signal-to-noise ratio with respect to the case of conventional microjunctions. The sharp apex of the cone determines a scanning nanocontact that, electrically, is correctly described by the so-called point contact geometry [59, 60]. While reducing the contact size, this three-dimensional description of charge transport increasingly deviates from the one-dimensional SB model, already at a modest doping level in the semiconductor. The corresponding I-V curve reflects the specific dependence on the size and shape of the junction, while the thickness of the barrier no longer depends solely on the doping level. For these reasons, the current follows the local electronic structure on a spatial length of the same order. A characteristic length, to be compared with the diode size $d$, is $L_{c}=\left(2 \varepsilon_{r} \varepsilon_{0}\right.$ $\left.\left(V_{\text {built-in }}+V_{\text {bias }}\right) / e N_{e-}\right)^{1 / 2}=106 \mathrm{~nm}$ for our case, that marks the transition from the classical (microscale) to the point contact geometry regime (nanoscale) [60].

In particular, there are two peculiar phenomena of general character that control the charge transport in a nano-sized junction that have to be taken into account. First, the full depletion approximation is no longer valid [61], since in this case the depleted volume, responsible for charge screening, tends to a spheroidal shape centered at the contact point. Consequently, this space charge 
(a)

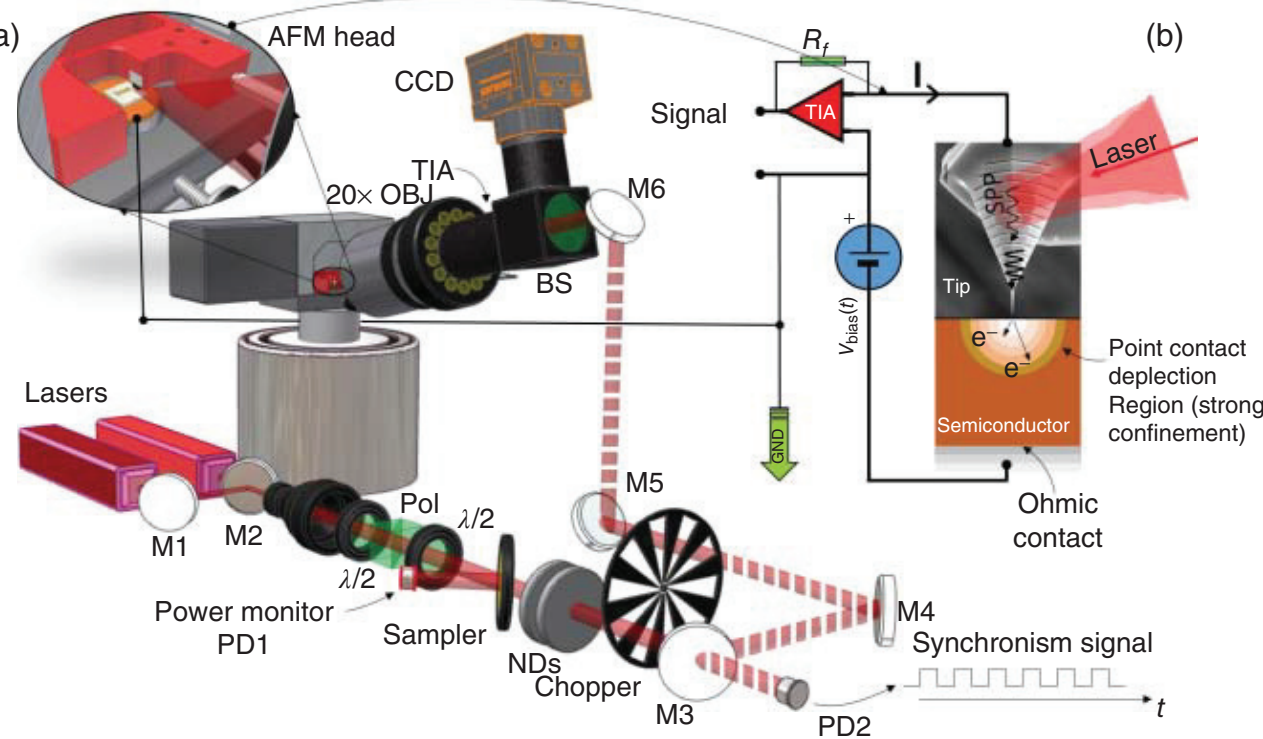

Figure 15.10 Experimental setup for photocurrent imaging. (a) Complete optical layout. Vertically polarized CW laser excitation at 670,980 , and $1060 \mathrm{~nm}$ (about $10 \mu \mathrm{W}$ of optical power on the coupling grating) is amplitude modulated with a mechanical chopper at $\sim 250 \mathrm{~Hz}$ and focused on the AFM tip. Additional elements are used to control the intensity and the polarization of the incident radiation. A transimpedance amplifier (TIA) amplifies the junction current. An external circuit is used to bias the junction to obtain the I-V curves, while a lock-in amplifier and a data acquisition (DAQ) board are used to acquire and record the signals. A confocal CCD allows a real-time monitoring of the scanning and illumination conditions. Inset: close-up image of the sample area. (b) Basic circuit scheme.

limited regime modifies deeply the field potential and controls the dynamic current performances. The second critical point concerns the assumptions of charge distribution continuity and homogeneity in the depleted region. Here, the meaningful length scale is the average distance among dopants, $N_{d}^{-1 / 3}$. In the cases reported below, $N_{d}$ is $\sim 1.1510^{17} \mathrm{~cm}^{-3}$, (referring to measures in Figures 15.11 and 15.13) we calculate $N_{e_{-}}{ }^{-1 / 3}=23 \mathrm{~nm}$, resulting to be in the same scale of the point contact.

From an instrumental point of view, this localized charge transport enhances the sensitivity of the measure down to a single dopant component.

\subsubsection{Hot Electron Mapping}

In Figure 15.11 we report the results of Ref. [14] that show the topography and corresponding photocurrent maps on two different surfaces obtained by local anodic oxidation and ion implantation in an n-type GaAs sample, excited at 647 and $980 \mathrm{~nm}$. The oxide pattern is written by AFM local anodic oxidation in air using high field discharge at room temperature at controlled humidity ( $\sim 40 \% \mathrm{RH},+4 \mathrm{~V}$ sample bias and at $4 \mu \mathrm{m} / \mathrm{s}$ writing speed), in contact mode (set point $<10 \mathrm{nN}$ ). Topography and $h e^{-}$intensity maps are simultaneously recorded with the same plasmonic tip and are shown in the overlaid 3D measure in Figure 15.11c. The photocurrent image is obtained in contact mode and under $\mathrm{N}_{2}$ atmosphere $(\sim 5 \% \mathrm{RH})$. This measurement allows extracting the resolution 


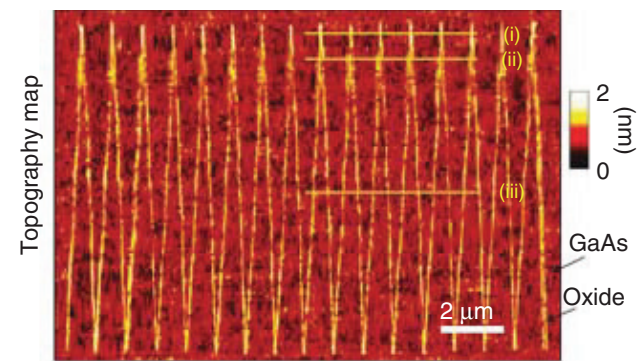

(a)
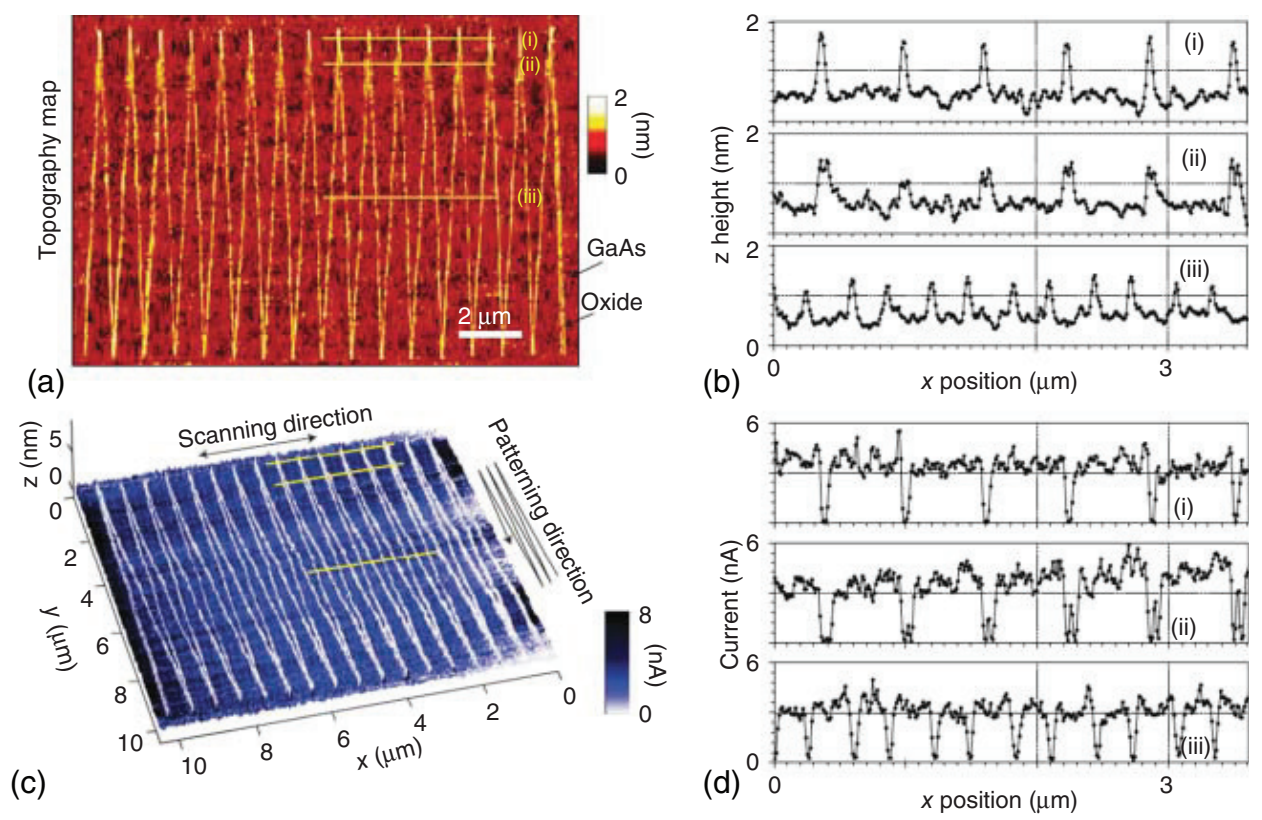

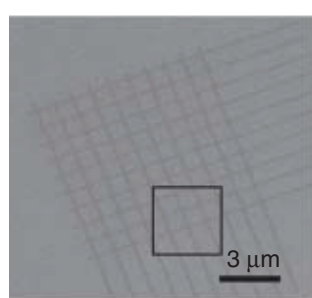

(e)

(f)

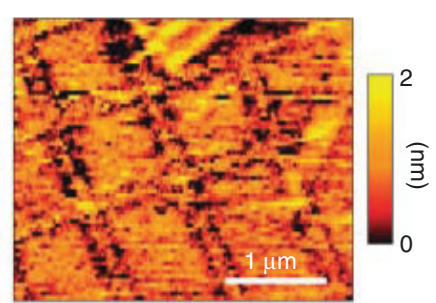

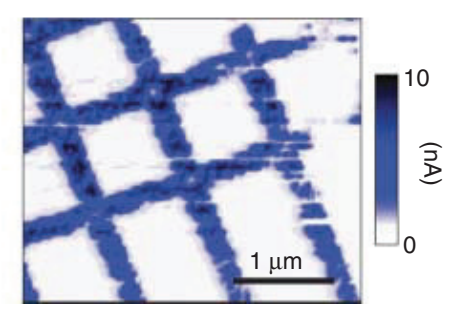

(g)

Figure 15.11 HENs maps of locally oxidized and ion-implanted patterned samples. (a, b) High-resolution AFM topography and height profiles of a continuous oxide pattern deposited on GaAs. The profiles (indicated by yellow lines in the topography map) are not deconvolved for the tip shape. (c) Photocurrent imaging overlaid on the 3D surface reconstruction shows simultaneously the current and topographic resolution. The zig-zag profile allows a direct check of experimental resolutions from line profiles (ii) of (b, d). (e) SEM image of the GaAs sample after implantation of $\mathrm{Ga}^{+}$ions. The black rectangle indicates the area relative to the topography and plasmonic he $e^{-}$maps, generated at $980 \mathrm{~nm}$ laser excitation $\left(E_{\mathrm{GAP}}>\right.$ $\left.1.26 \mathrm{eV}>e \Phi_{b}\right)$, shown in (f, g). Reprinted with permission from [14], copyright NPG 2013.

and sensitivity of the technique that can be routinely achieved, as can be inferred from the line profiles in the map. The reproducibility of the profiles in trace and retrace demonstrates the stability of this scanning probe technique. Figure $15.11 \mathrm{f}$ and g shows the topography and photocurrent maps of the GaAs substrate reported in the SEM micrograph of Figure 15.11e. In this case, we implanted $\mathrm{Ga}^{+}$ions in the surface by FIB, creating a grid pattern $(40 \mathrm{pA}, 100 \mathrm{~ns} / \mathrm{pt}, 30 \mathrm{keV}$, single pass). Using these fabrication parameters, the minimal dose of ions is used to locally modulate the electrical conductivity, with a negligible modification of surface topography, which remains essentially flat. The measure in Figure $15.11 \mathrm{~g}$ reveals a conductance cross section of the implanted line, which reflects the typical spreading of implanted dose. We remark that this measure was performed at $980 \mathrm{~nm}(1.26 \mathrm{eV})$, for which a photovoltaic contribution for the GaAs is not expected, being the excitation energy smaller than the bandgap $\left(E_{\mathrm{GAP}} \approx 1.45 \mathrm{eV}\right.$, 
Si doped n-type GaAs, $N_{d} \sim 10^{17} \mathrm{~cm}^{-3}$ ). Finally, we stress that for both samples the standard conductive electric map does not reveal any special feature within the sensitivity limits of the technique.

\subsubsection{Hot Electron Resolution Limit}

To quantify the ultimate spatial resolution and sensitivity of the technique we have prepared an ad hoc sample. An n-type GaAs substrate was spin coated with a thin poly(methyl methacrylate) (PMMA) layer $(<40 \mathrm{~nm})$ and subsequently patterned by means of electron beam lithography in aligned lines of variable width (from 70 to $100 \mathrm{~nm}$ ), and interdistances of $30 \mathrm{~nm}$. An overexposure and a controlled developing process allowed obtaining a polymer thinning over the whole area. The process resulted in a resist coverage with randomly dispersed nanometer-sized holes generally less than $1 \mathrm{~nm}$ in depth, where the substrate is accessible. This sample allows testing the experimental resolution limit by measuring the point spread function for hot electron injection through a SB.

The sample was scanned in small areas at high spatial resolution. The $h e^{-}$map of Figure 15.13d clearly reveals repeated lines and holes features. To test the reproducibility, the $h e^{-}$current was acquired both during trace and retrace pass while scanning the surface.

To correctly estimate the ultimate spatial resolution achievable at usual speeds, we switched from a lock-in amplifier (LIA) detection scheme, requiring an average over several optical cycles (introducing an additional broadening $\tau_{\mathrm{LIA}}=30 \mathrm{~ms}$ $\sim 9$ optical cycles), to a post-processing analysis of the current data recorded at a high sampling rate. After a spectral analysis, we applied a digital infinite impulse response (IIR) low-pass filter with about $1 \mathrm{kHz}$ bandpass to remove spurious high-frequency noise from the signal. Then, as shown in Figure 15.13b and c,
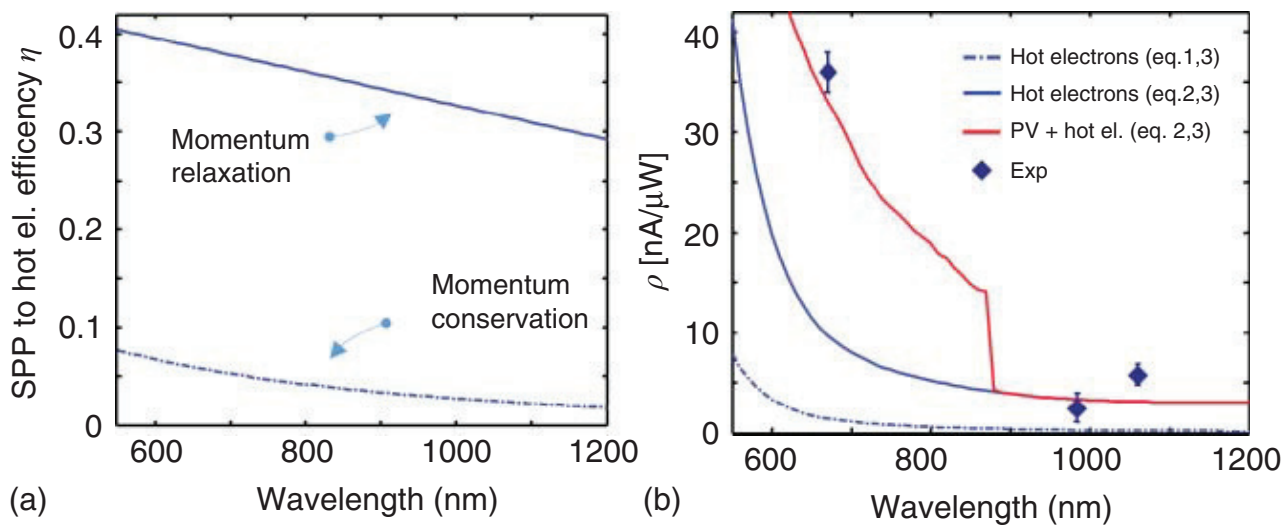

Figure 15.12 (a) Calculated transmission efficiency $\eta$ of the $h e^{-}$across the $n$-type GaAs/Au Schottky junction as a function of wavelength. The two curves refer to the different limit of the momentum conservation and relaxation. (b) Experimental device responsivity $\rho$ (blue diamond) at 670, 980, and $1060 \mathrm{~nm}$ and its theoretical values, as calculated from Eq. (15.8). The two momentum exchange regimes generate the blue dotted line, Eq. (15.6), and blue continuous line, Eq. (15.7). The red line adds the photovoltaic contribution, as obtained from literature data, to the responsivity calculation of Eq. (15.8) combined to Eq. (15.7). Adapted with permission from [14], copyright NPG 2013. 
the current was reconstructed point by point as $\left\langle I_{\text {on }}\right\rangle-\left\langle I_{\text {off }}\right\rangle$, for each optical period. The use of this matched filter allows for a further increase in $S / N$ ratio, maintaining the time resolution and giving the true spatial resolution while scanning at a reasonable speed. We selected the values to be averaged (colored point
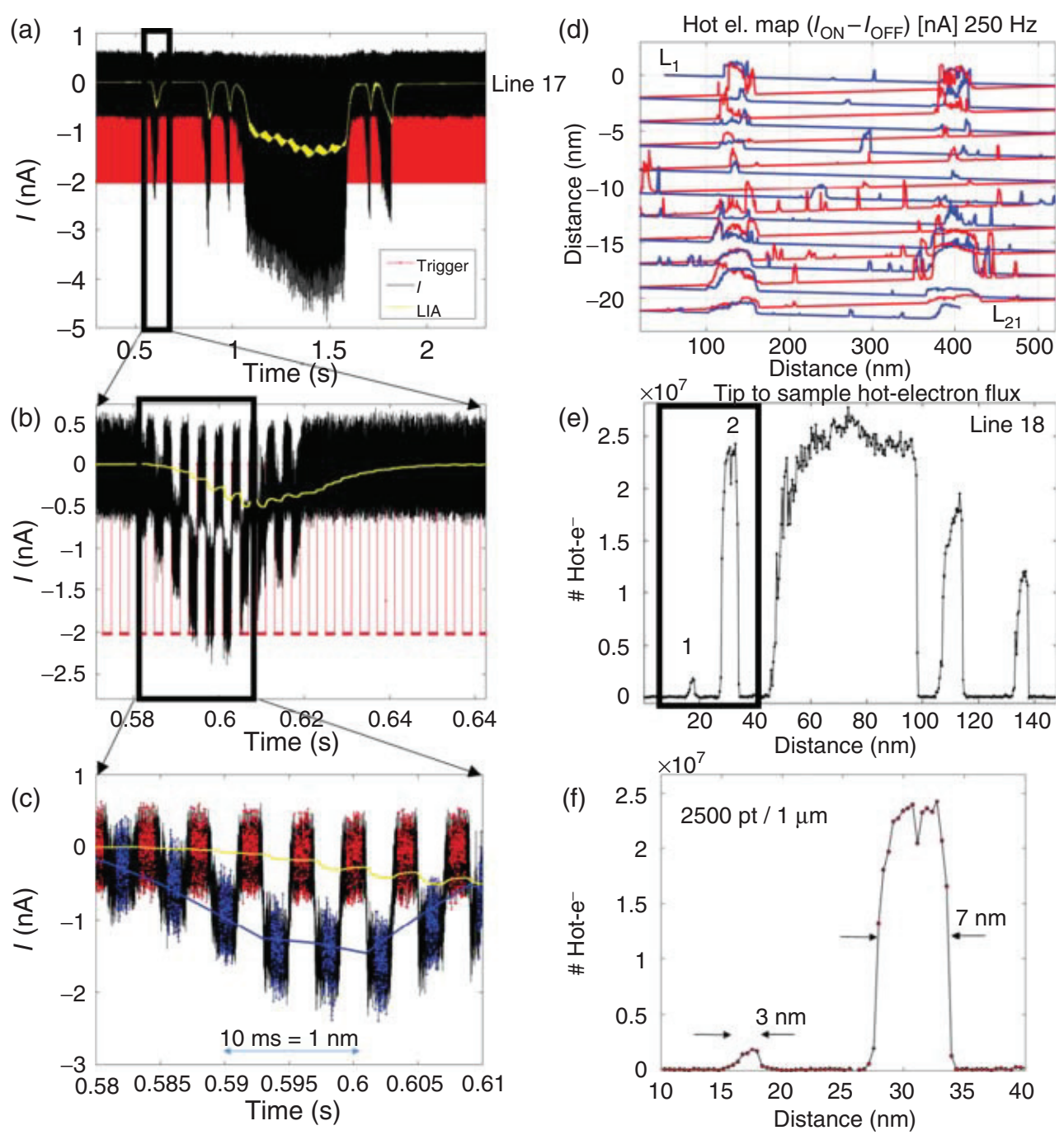

Figure 15.13 Experimental resolution. (a) A 2 s record of the current stream while scanning the patterned n-type GaAs/Au junction, at $670 \mathrm{~nm}$. (b) The graph refers to an acquisition time of $0.1 \mathrm{~s}$. Here we show on and off states on the current flow (black line) and the laser trigger events (red lines). The yellow line, reported for the sake of comparison, is the output signal of the lock-in. (c) Few optical cycles of acquisition time. Here we have indicate the data points selected to calculate the $I_{\text {on }}$ (blue) and $I_{\text {off }}$ (red) local values as described in the text. The blue broken line connects consecutive points from averaged cycles, demonstrating the optimal reconstruction of the temporal profile even for high-dynamic signal, differently from the slower LIA detection procedure. (d) $\left\langle I_{\text {on }}\right\rangle-\left\langle I_{\text {off }}\right\rangle$ surface reconstruction. Blue and red lines display AFM trace and retrace, demonstrating a correct agreement. The first $\left(\mathrm{L}_{1}\right)$ and the last $\left(\mathrm{L}_{21}\right)$ lines of this reconstruction are indicated. (e) Detail of the spatial reconstructed signal of line $L_{18}$, in terms of number of $h e^{-}$per pixel. The detail of the first and second peaks, reported in (f), allows the direct estimation of the ultimate experimental resolution, as FWHM of peak 1 or as $10-90 \%$ of the rising front of peak 2 , which is $3 \mathrm{~nm}$. 
in Figure 15.13c) by excluding the transient regimes in the signal, synchronizing it with the modulated laser intensity. In this way, we were able to pin down a point every $0.4 \mathrm{~nm}$, cutting out almost all the noise contribution while preserving the maximal part of the signal. Figure $15.13 \mathrm{e}$ and $\mathrm{f}$ demonstrates an ultimate full-width-at-half-maximum (FWHM) resolution of $3 \mathrm{~nm}$, preserved over repeated lines during both trace and retrace.

\subsection{Kelvin Probe, HENs, and Electrical Techniques}

In this section, we compare the technical advances proposed by HENs in terms of chemical sensitivity and spatial resolution, also in comparison with other electrical applications of AFM microscopy, such as scanning Kelvin probe microscopy (SKPM), sensitive to the same parameters, and conductive AFM. Further references to these last techniques can be found in more detail in a dedicated chapter on the topic.

\subsubsection{SKPM Theoretical Frame: a Short Introduction}

The most diffused technique providing a direct measurement of the contact potential between two surfaces is referred to as Kelvin probe, after its inventor Lord Kelvin, who first postulated this method in 1861. The first implementation on an AFM setup, by employing a metallic tip at the end of a cantilever, dates back to 1991 [62], when a nanometric spatial resolution $(50 \mathrm{~nm})$ with a remarkable sensitivity $(0.1 \mathrm{~V})$ was first demonstrated. Since then, continuous efforts to improve both parameters to their ultimate extent have been made.

The built-in electric field between a sample surface and a reference electrode depends on many physical properties of the materials. A major contribution is given by the chemical potential mismatch resulting from the difference between the Fermi and the vacuum level in the two materials when they are electrically connected to create a junction.

The energy required by electrons to be extracted from a material is given by

$$
\phi=E_{\mathrm{Vac}}-\varepsilon_{F}
$$

where $E_{V a c}$ is the vacuum. At thermal equilibrium, the Fermi levels of two electrically connected materials pair, giving origin to a potential difference

$$
-e \Delta V_{\mathrm{CPD}}=\phi_{1}-\phi_{2}=E_{\mathrm{Vac} 1}-E_{\mathrm{Vac} 2}=-e\left(V_{1}-V_{2}\right)
$$

which represents the work done to bring an electron from one side of the junction to infinity and back to the second side.

By adding an external voltage $V_{\text {ext }}=V_{\mathrm{DC}}+V_{\mathrm{AC}}\left(\omega_{\mathrm{el}}\right)$, it is possible to measure the capacitive coupling between the two electrodes, synchronously measuring macroscopic properties such as the transport current or the electrostatic force. Minimal coupling will occur when $V_{\mathrm{DC}}=V_{\mathrm{CPD}}$, therefore, by externally tuning a DC bias, it is possible to measure the contact potential between the two electrodes. In AFM, the cantilever already provides an extremely sensitive force sensor [63], which can be used to measure the capacitive force. 
The measurement technique relies on the following strategy: line by line the surface is scanned two times. During the first pass, a topography map is acquired in AC mode to get the morphology of the surface. In the second one, the same line is repeated at a different height $z^{\prime}(x, y)=z_{\text {topo }}(x, y)+\Delta z$ while applying $V_{\text {ext }}$ to the tip.

The resulting tip-sample force is

$$
F_{\text {tot }}\left(z^{\prime}, t\right)=k\left(z(t)-z^{\prime}\right) \sin (\omega t)-F_{\text {Pauli }}-F_{\text {vdW }}-\frac{1}{2} \frac{\mathrm{d} C}{\mathrm{~d} z}\left(V_{\text {ext }}-V_{\mathrm{CPD}}\right)^{2}
$$

where $F_{\text {Pauli }}$ and $F_{\mathrm{vdW}}$ are the short-range repulsive (topography) and long-range attractive van der Waals forces, respectively, and $k\left(z(t)-z^{\prime}\right)$ is the withdrawal force due to the cantilever elastic constant $k$ for an oscillation amplitude $A=z(t)-z^{\prime}$. The last term is the capacitive coupling given by the total tip-sample voltage, which brings in the contact potential. During the second pass, the tip voltage is modulated by $V_{\mathrm{AC}}$ to induce a tip oscillation at a frequency $\omega_{\mathrm{el}}$ (usually slightly different from the mechanical resonance frequency of first pass) with oscillation amplitude $A_{\mathrm{el}}$ that can be remarkably different from the first pass one. A proper second pass elevation $\Delta z$ allows to cancel the Pauli contribution and to maximize the sensitivity to the capacitance coupling with respect to the van der Waals forces at the same time.

The capacitive force can be rewritten as

$$
\begin{aligned}
-\frac{1}{2} \frac{\mathrm{d} C}{\mathrm{~d} z}\left(V_{\mathrm{ext}}-V_{\mathrm{CPD}}\right)^{2}=-\frac{1}{2} \frac{\mathrm{d} C}{\mathrm{~d} z} & {\left[\left(V_{\mathrm{DC}}-V_{\mathrm{CPD}}\right)^{2}+2\left(V_{\mathrm{DC}}-V_{\mathrm{CPD}}\right)\right.} \\
\times & \left.V_{\mathrm{AC}} \sin \left(\omega_{\mathrm{el}} t\right)+\frac{V_{\mathrm{AC}}^{2}}{2}\left[1-\cos \left(2 \omega_{\mathrm{el}} t\right)\right]\right]
\end{aligned}
$$

This has three main components in the force frequency spectrum, one DC, one at frequency $\omega_{\mathrm{el}}$, and one at $2 \omega_{\mathrm{el}}$. An additional feedback loop adjusts $V_{\mathrm{DC}}=V_{\mathrm{CPD}}$ to cancel the force component at $\omega_{\mathrm{el}}$ point by point, providing a local measurement of the contact potential. At the same time, the capacitive coupling $-1 / 2 \frac{\mathrm{d} C}{\mathrm{~d} z} V_{\mathrm{AC}}^{2}$ can be also measured from the second harmonic, obtaining the scanning capacitance signal, which is a widely used technique in microelectronics to estimate surface oxidation states.

For a direct comparison with HENs, we have performed a measurement that provides the resolution achievable with SKPM on a sample like the one used for the data in Figure 15.11.

A new GaAs (n-type $N_{d}=10^{16}$ ) substrate was freshly cleaned in $\mathrm{HCl} 37 \%$ before the nano-oxidation procedure. We used commercial PtIr-coated Asylum electrilever $(K=2 \mathrm{~N} / \mathrm{m}$, nominal tip radius $=30 \mathrm{~nm}, 75 \mathrm{kHz})$ on an Asylum MFP-3D AFM microscope. Writing parameter and experimental condition were $9 \mathrm{~V}$ sample bias, oxidative current $3 \mathrm{nA}$, and writing speed $500 \mathrm{~nm} / \mathrm{s}$ in environment humidity of $45 \%$. The oxidized pattern was then read by scanning at $90^{\circ}$ the surface. The results of topography and SKPM potential map $5 \times 5 \mu \mathrm{m}^{2}$ area are reported in Figure $15.14 \mathrm{c}$ and d, with details of a smaller scan in 
(a)

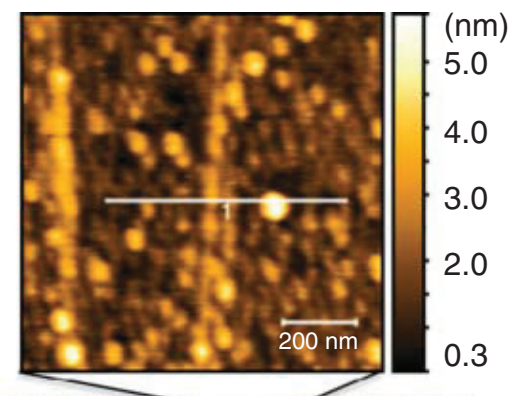

(c)

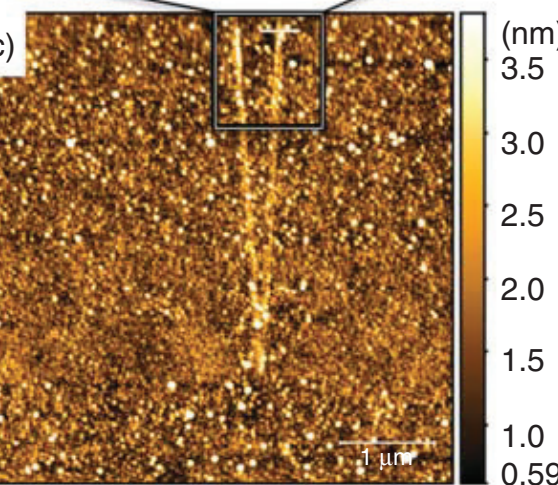

(b)
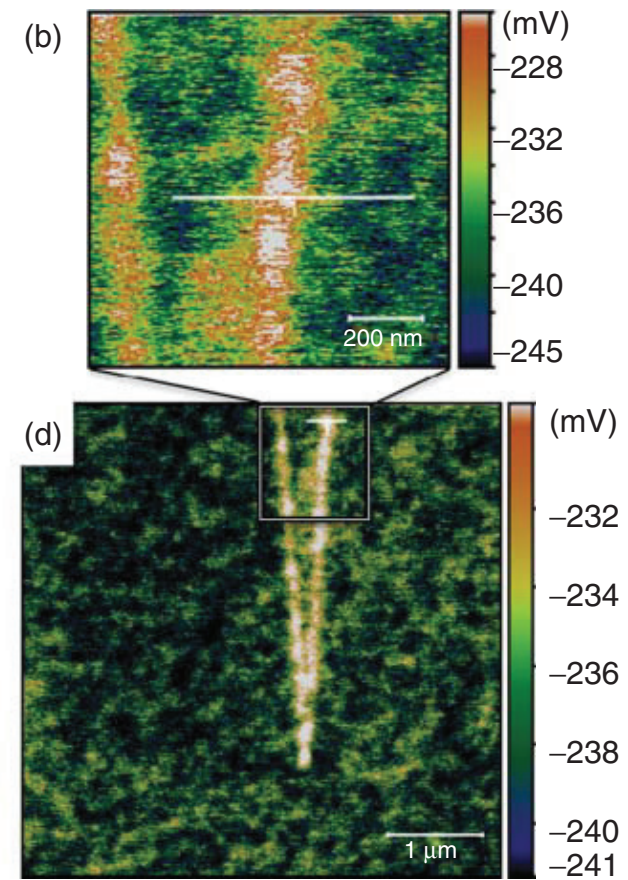

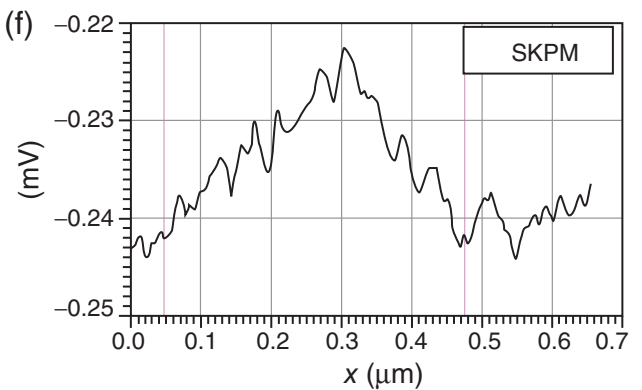

(e)

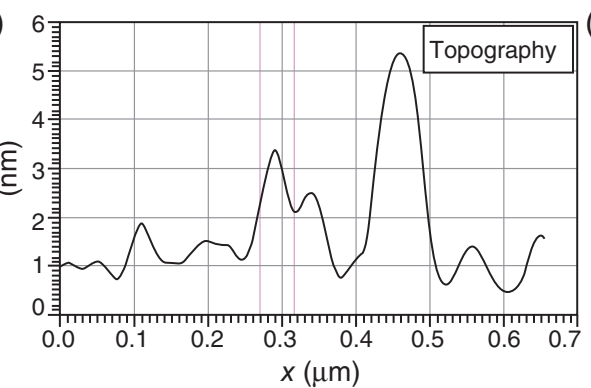

Figure 15.14 SKPM characterization of an oxide-patterned GaAs sample (n-type $N_{d}=10^{16}$ ) with nanoxidtive regime at $9 \mathrm{~V}$ sample bias and oxidative current $3 \mathrm{nA}$ in relative environment humidity of $45 \%$. (a, b) Detail images of the topography and SKPM maps shown in $(c, d)$. Here topography reveals features not accessible with SKPM. $(e, f)$ The graphs report the line profiles indicated with white lines in the zoomed area.

Figure 15.14a and b. From the line profiles of Figure 15.14e and f, corresponding to the high-resolution zoomed areas Figure 15.14a and b, it is possible to evaluate the spatial resolution of the technique with respect to the topography one. The not deconvoluted topography reveals features as large as $46 \mathrm{~nm}$, measurable between markers in Figure 15.14e, while SKPM results are limited to a line resolution not better than $200 \mathrm{~nm}$.

This technique has many drawbacks and implications:

1) The sensitivity of the measurement strongly depends on the distance $\Delta z$ set during the second pass. For best resolution and sensitivity, since the closest tip-sample distance should be $\Delta z-A_{\mathrm{el}}>0, A_{\mathrm{el}}$ is usually by far lower than $A_{\text {topo }}$, and both tip radius $R$ and $z$ depend through the relation $A_{\mathrm{el}}=\frac{V_{\mathrm{AC}}}{2 k} \frac{\mathrm{d} C(R, z)}{d}$, the optimal distance has to be tuned during the measurements, also with $\Delta z<0$. 
2) A remarkably high resolution and surface sensitivity can be achieved in frequency modulation mode. This is due to the reduced oscillation amplitude allowing a second pass a few nanometers off the surface. This is commonly performed in an ultrahigh vacuum (UHV) environment where such a technique is more stable [64]. However, the UHV environment is usually less compatible with biological samples, reducing the field of application for this technique.

3) A tip modification, usually occurring during first pass due to the contact with the sample, changes its surface potential. This affects the final results in three different ways:

a) The tip can pick up tiny amounts of materials from the sample (mostly occurring for soft/organic specimens), changing sensitively the reference work function during a scan.

b) Due to environmental humidity and the applied voltage, the tip can oxidize.

c) The tip can modify its shape by plastic deformation or material loss. This, again, affects the results by changing tip radius, that is, capacitance, and, to a minor extent, by redefining local work function due to different geometry. This effect can be important in case of heavy damage to coated tips, when the metal is peeled off, leaving the silicon substrate exposed.

4) As discussed, $C$ depends on $z$ and $R$ in the first approximation. To be more precise, one should consider the true shape of the electric field between tip and sample giving $\boldsymbol{F}(x, y, z)=-e \boldsymbol{E}(x, y, z)=e \nabla V$, which more properly accounts for the tip and sample geometries and compositions. The shape of the electric field $\boldsymbol{E}$ gives the effective resolution and sensitivity, worsened by the spreading of the field at the sample surface.

5) The material of the tip also technically plays an important role: aside from high conductivity, geometrical and chemical stability imposes main requirements on the tip. For these reasons Pt-, PtIr-, PtSi-, or Ir-coated silicon tips are widely used, ending up with a tip radius usually around $30 \mathrm{~nm}$. For sharper doped Si tips, the additional oxidation effects have to be taken into account. For biological materials, a good choice would rely on hydrophobic tips, such as conductive PtC-coated ones, that are less prone to material pick up due to hydrophobic/hydrophilic repulsion.

6) Moreover, SKPM is an inherently surface-sensitive technique. The dependence on surface contaminations and charge distribution cannot be neglected. Due to the presence of fixed surface charges in trap states or embedded in native oxide on top of the sample surface, an additional potential appears as an offset on the $V_{\mathrm{CPD}}$ as $V_{q}=q N_{F} t_{\text {ins }} / \varepsilon_{\text {ins }}$. Here, $q$ is the elementary charge, $N_{F}$ is the charge density, $t_{\text {ins }}$ is the thickness of the insulating layer separating the tip from the substrate, and $\varepsilon_{\text {ins }}$ is the average dielectric constant. Assuming a surface charge in the order of $N_{F}=10^{10}-10^{11} \mathrm{~cm}^{-2}$, values which are commonly achieved for high-quality microfabrication oxide, one gets $V_{q}=5-50 \mathrm{mV}$ which is a relatively high value for the offset in the measurements.

7) Tip oxidation and contamination have to be considered. Pre- and after-measurement calibrations on a reference sample, such as freshly cleaved highly oriented pyrolytic graphite, have to be routinely employed for accurate measurements [65]. 


\subsubsection{HENs}

As introduced before, HENs is a charge transport AFM technique performed in contact mode. The net charge transfer is strongly dependent on the initial photon energy, on the metal of the tip, and on the local electronic properties of the sample at the nanometer scale. Here we compare HENs to different AFM electrical modes in terms of spatial resolution, sensitivity, and dynamics.

\subsubsection{Spatial Resolution}

HENs provides a contrast on the local Fermi level of the tip and the conduction band of the sample and its local modification due to adsorbed materials, surface charge, and doping, but with a remarkably higher spatial resolution. The reason is that while in SKPM the final resolution is affected by the second pass elevation $\Delta z$ giving the relatively high broadening of the electric field at the surface, in HENs the final resolution is practically limited just by the area of the junction. In contact mode, the effective tip-sample contact can be limited to a few nanometers in diameter, similar to the case of conductive AFM and scanning tunneling microscope (STM) in air. The main difference is that no external polarization bias is needed to drive the current since the plasmon compression gives the energy and the momentum to overcome the $\mathrm{SB}$ at the interface. In contact AFM or STM, an external bias of a few Volts can easily generate an electric field exceeding the dielectric breakdown of nanomaterials at the interface [66], and induce oxidation and/or metal electromigration from the tip. This results in both tip and sample modification, as well as junction instability, phenomena frequently occurring while operating under environmental conditions. Apart from these practical aspects, adiabatic compression of evanescent plasmonic modes provides a strong localization of the electric field at the tip, stronger than the quasi-static field other electrical AFM modalities rely on. The electromagnetic simulations in Figure 15.15 show how in a plasmonic tip the electric field is distributed around the apex. Here the field is naturally concentrated in a spatial region limited to a few nanometers around the tip, different from the quasi-static case, where a relatively high broadening is given by $E=-\nabla V$. Then in HENs, the point contact area is further localized in the junction region once the contact is established.

\subsubsection{Sensitivity and Specificity}

In HENs, the generated hot electrons bring the SPP momentum associated to the adiabatic compression. This allows them to be selectively injected into the semiconductor with efficiency depending on crystallographic directions. Another important difference is in the energy distribution of the carriers involved in the process. In conductive AFM and STM/STS spectroscopy, for instance, by applying an external voltage the Fermi level of the tip is aligned with different energy states of the substrate. The current comes from the facing of occupied states on the tip and empty states on the sample or vice versa, allowing tuning the current and its direction by means of an external bias. By increasing the bias, the number of states entering in the conduction process increases, involving states even many electronvolts far from the Fermi level, whose number 
Electric field norm $(\mathrm{V} / \mathrm{m})$

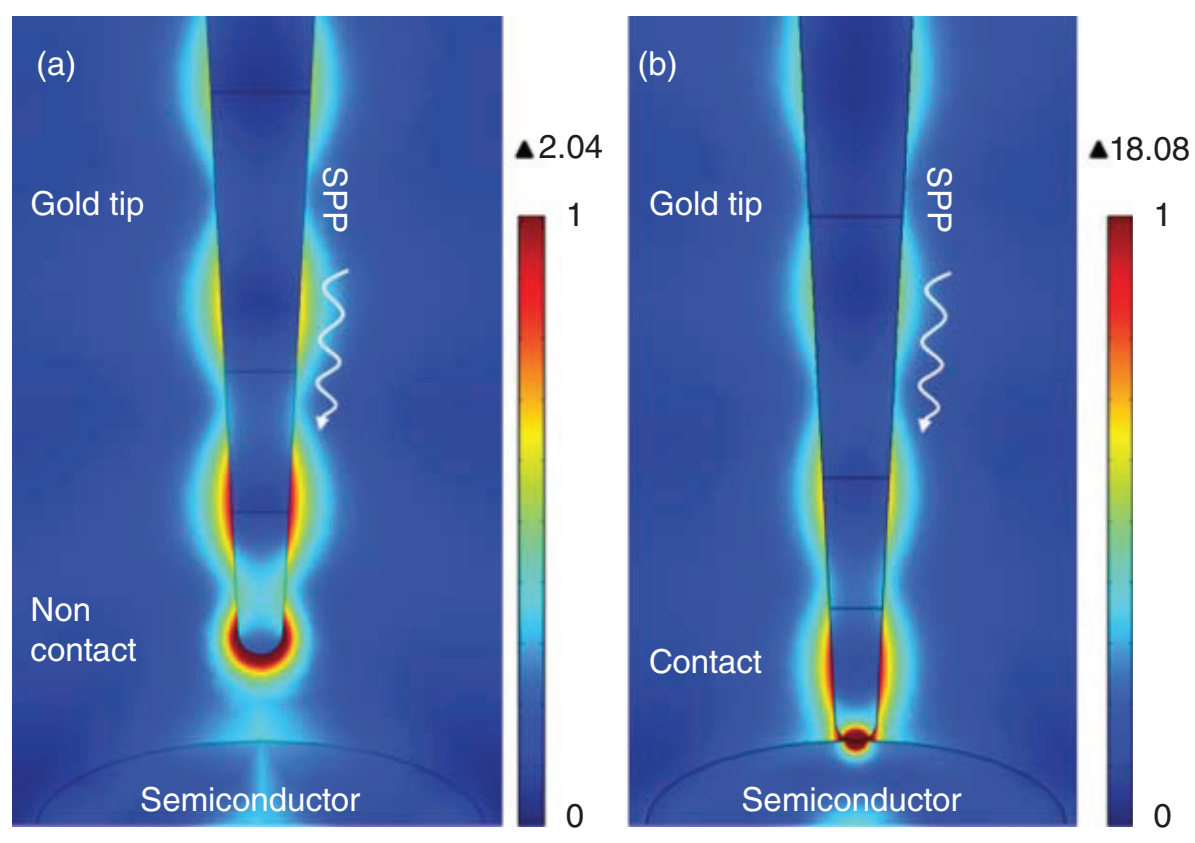

Figure 15.15 Electric field norm of a TM0 mode along the conical tip $\left(R_{\text {tip }}=25 \mathrm{~nm}\right)$, excited at $670 \mathrm{~nm}$. (a) Simulation results for the case of no contact between the metal tip and the semiconductor. (b) Tip in contact with the semiconductor. A further enhancement of the induced adiabatic SPP focusing at the tip apex is observed. In both cases, fields are normalized to the input amplitude, $E_{0}=1 \mathrm{~V} / \mathrm{m}$. The numerical simulation clarifies the effect of the contact on the plasmonic field at the tip apex. Higher field enhancement at the Au tip is observed when the contact is established, due to the high value of the refractive index of the semiconductor ( $n \sim 4.4$ in our case). Reprinted with permission from [14], copyright NPG 2013.

is given by the integral of the occupancy probability over the allowed energies $P(E) \propto D(E-e V) f(E-e V)$ (D and $f$ are the density of states and the Fermi distribution). Since the density of states in metals is described by a quite broad distribution, the number of electrons contributing to conduction increases with $\mathrm{V}$ as the integral over the states. The two current spectroscopies, I-V and the more energy-resolved $\frac{\mathrm{d} I}{\mathrm{~d} V}$, allow to evaluate the bandgap, the energy levels distribution, and the gaps between the highest occupied and lowest unoccupied molecular orbitals in materials and molecules by probing them with a continuum of energy states of the tip up to the Fermi level. Similarly, the applied voltage in SKPM mode is optimized to adjust the Fermi levels in the two materials (tip and sample), providing the measure of the contact potential. In both cases, the electrons at the Fermi level, which by definition is an equilibrium state, play a key role in the measurements. On the other hand, HENs relies on hcs, which by definition are nonequilibrium (excited) states in electronic distribution. In this process the decay of a plasmon produces hcs in excited states, whose occupancy probability and energy distribution $P(E)$ can be calculated following the work of Gong and Munday [57] as

$$
P(E) \propto D\left(E-E_{\mathrm{Ph}}\right) f\left(E-E_{\mathrm{Ph}}\right) D(E) f(E)
$$


According to this formula, an electron in the half-filled band can be excited up to an energy $E$ which is set experimentally by the light source. This produces a hot electron-hole pair, whose energy distribution is reported in Figure 15.9 for the most commonly used plasmonic metals and energies.

These results allow for some considerations:

1) Selecting the external energy, that is, laser wavelength, it is possible to control the $h c$ energy density in the metal to have a sharp peak overcoming the junction barrier. In this case, only a narrow distribution of excited states contributes to the electronic transport and probes the energy states of the sample. This goes toward a highest energy resolution for spectroscopy studies and energy-resolved imaging.

2) The basic process of charge transfer can occur at zero bias. This allows testing energy levels without an external DC electric field that, for standard conductive sharp tips very close to the surface, can easily exceed an electrical field of $10^{9}-10^{10} \mathrm{~V} / \mathrm{m}$. This is believed to be a great advantage for many samples, such as thin oxidation layers, heterojunctions, and biological molecules since no perturbation or shrinking is induced. This is a very promising aspect when Raman measurements are combined with current transport measurements in a multiprobe configuration.

3) In HENs, the addition of a small modulation voltage can be used to refine the local density of states measurement of the sample by tuning the energy of the hot charges. This technique therefore could be used in parallel with other electrical modes such as SKPM or STS.

4) As shown in Figure 15.9 and further explained in the literature [21, 57], the transport is due not only to hot electrons but also to hot holes, since the plasmon decays into a carrier pair. According to the material and the incident photons, either hot electron or hot hole current can prevail. This second case, for instance, occurs for $\mathrm{Cu}$ and $\mathrm{Au}$ for both excitation wavelengths of $\lambda=700$ and $400 \mathrm{~nm}$ and for $\mathrm{Ag}$ at $\lambda=400 \mathrm{~nm}$ [21]. This opens up additional possibilities for spectroscopies.

5) A shaping of electron density can be achieved in HENs also with the combination of different excitation wavelengths at the same time.

6) In the classical electrical mode, the detectable dynamics is usually quite limited (well below megahertz range). HENs process is triggered by laser excitation and the mechanism underneath is intrinsically a nonequilibrium one, hence the dynamics can be pushed to the very limit of the state-of-the-art detection systems for current acquisition (trans-impedance amplifiers).

7) According to recent literature [57, 67], new material can be explored to realize plasmonic tips, offering the possibility of optimizing and tuning HENs for different applications. Remarkably, most of them offer mechanical properties similar to the ones commonly used for electrical tip coatings (Young Modulus $E_{Y} \approx 60-130 \mathrm{GPa}$ ), appearing therefore well suited for contact probes fabrication. 


\subsection{Fast Pulses in Adiabatic Compression for Hot Electron Generation}

One of the trends that has emerged in recent years among the applications of SPP adiabatic compression in plasmonic nanocones is the use of these tools in combination with pulsed lasers to access a faster dynamic. Pulsed laser sources are already fundamental in many fields of research as well as in many industrial, medical, and biological applications. Nevertheless, their use in microscopyand microscopy-related applications remains limited by diffraction phenomena when combined with far-field focusing tools as microscope objectives. Coupling with modes that experience adiabatic compression in a plasmonic tapered waveguide is certainly a promising strategy to overcome this limitation. This type of application has already been explored in literature and the possibility of coupling ultrashort laser pulses in metallic cones squeezing the optical excitation to a nanometric-sized spot has been previously demonstrated $[68,69]$.

The interest in this particular application, due to the fact that it is possible to achieve nanometric-size excitation, derives from the possibility of having both high peak intensity and time-resolved excitation, hence leading to the long-searched-for goal of a coherent control of electromagnetic field at nanometer-large and femtosecond-limited scale [70].

A major concern which arises while dealing with ultrashort laser pulses in optical media, is the time broadening and pulse reshaping due to group delay dispersion that different frequencies accumulate over propagation in any dispersive medium. This does not seem to be a major concern in plasmonic propagation in the adiabatic compression regime, since an electromagnetic pulse propagating along a metallic nanocone experiences modest group velocity dispersion and hence travels with an almost unperturbed temporal profile $[68,69]$.

Particular attention, in this case, has to be paid to the coupling mechanism. In previous paragraphs, we described the main excitation techniques that we use to convert photons into surface-bounded plasmons. The different techniques would provide different results in the case of ultrafast excitation for both what concerns coupling efficiency and for what concerns the time dispersion. Direct coupling of radially polarized light beams at the base of a metallic cone seems to be a promising way to control pulse distortion. This method is weakly sensitive to wavelength spreading, resulting in being weakly affected by the broad spectrum of the light source. Simulations on this kind of structure indicate a substantial preservation of the laser pulse after propagation along a 10- $\mu \mathrm{m}$ gold cone [33] and that this coupling efficiency can be extremely effective, even approaching 90\% [71].

Coupling through a grating, on the other side, has a natural limit, as it is intrinsically wavelength dependent. Many authors, anyway, explored this kind of configuration, since it happens to be the most practical one in many experimental arrangements. They managed to find interesting solutions to compensate for the large spectral range covered by these excitation sources, 
mostly based on the use of linearly chirped gratings [68, 72]. Nevertheless, it has been demonstrated that coupling of 10-fs laser pulses onto a gold nanocone via a normal nonchirped grating preserves substantially unchanged the pulse time-frequency profile. Also, in this case, numerical analysis demonstrates the poor distortion introduced by the grating itself, and the authors were able to measure directly the frequency-resolved autocorrelation of the plasmonic pulse at the apex of the nanocone [69].

Applications of nanofocusing of pulsed lasers are still at a very early stage. Efficient nano-localized second-harmonic generation [68], nonlinear optical measurements [73], and high-harmonic generation in tapered metallic hollow conical waveguides [18] have already been demonstrated. The possibility of extending time-resolved techniques down to nanoscale opens up countless potential applications.

Another outcome is the inherent enhancement of the electromagnetic field at the cone apex, which triggers promising applications of nonlinear optics in fields such as nanoimaging and nanocharacterization. Tip-enhanced electromagnetic field with high spatial and temporal resolution has already demonstrated to provide insight into infrared molecular spectroscopy [74]. The application to nonlinear Raman [75-78] of adiabatically compressed pulsed excitation will certainly represent a significant improvement to imaging techniques at the nanoscale, particularly in the field of biology and bio-imaging.

With respect to $h c$ generation, application of pulsed laser to metallic cones represents an interesting perspective from different points of view. From one side the possibility of performing time-resolved nonlinear characterization in parallel to $h c$ measurements and optical spectroscopies, such as enhanced Raman, paves the way for parallel multiprobe nanosized characterization of the sample. Time-resolved $h c$ injection could have interesting applications in understanding important phenomena like zitterbewegung in two-dimensional material $[79,80]$ and nonlinear plasmon-induced photocatalisys [81]. Moreover, the possibility of obtaining free-space electron emission from excited cones in adiabatic compression, exploiting multiphoton absorption of ultrashort pulses to overcome the metal work function has been recently demonstrated [82].

\subsection{Conclusion}

In this paper, we revised our recent results on hot electron SPM showing also our ultimate spatial resolution limit of $3 \mathrm{~nm}$ and compared them with the literature to give perspectives and possible additional implications. In our research, we are aware of the unicity of our approach and having in our hands a novel multipurpose method for investigating materials at nanoscale and to femto-attosecond dynamics. In the past years we demonstrated the full compatibility of adiabatic compression with AFM techniques. Now our effort is devoted to merge AFM force spectroscopy, light-enhanced spectroscopy, and hot electron spectroscopy. Applied to molecules, we foresee the possibility of obtaining novel structural information in a wide range of environmental conditions never explored before. 


\section{Acknowledgments}

We thank our colleagues at BIONEM Lab of Magna Graecia University, Catanzaro, and former colleagues at Nanostructures Division of IIT, Genoa, Italy, for the contribution they have given to this research activity in the past years.

We acknowledge financial support from (i) King Abdullah University of Science and Technology for OCRF-2014-CRG grant, (ii) the Italian Ministry of Health under project nos. GR-2010-2320665 and GR-2010-2311677, and (iii) the European project EUROMBR (grant no. 608104).

\section{References}

1 Shahbazyan, T.V., Stockman, M.I. (2014) Plasmonics: Theory and Applications, Springer Netherlands.

2 Dragica Vasileska, S.M.G. (ed.) (2011) Nano-Electronic Devices, Nano-

Electronic Devices. Semiclassical and Quantum Transport Modeling, Springer, New York.

3 Xie, Z., Yu, W., Wang, T., Zhang, H., Fu, Y., Liu, H., Li, F., Lu, Z., and Sun, Q. (2011) Plasmonic nanolithography: a review. Plasmonics, 6, 565-580.

4 Kumar, R. and Kumar, N. (2014) Nanotechnology and Nanomaterials in the Treatment of Life-threatening Diseases, Elsevier Inc., 225 Wyman Street, Waltham, MA.

5 Binnig, G., Quate, C.F., and Gerber, C. (1986) Atomic Force Microscope. Phys. Rev. Lett., 56, 930-933.

6 Kalinin, S.V., Gruverman, A. (2011) Scanning Probe Microscopy of Functional Materials. Springer.

7 Anderson, M.S. (2000) Locally enhanced Raman spectroscopy with an atomic force microscope. Appl. Phys. Lett., 76, 3130-3132.

8 Schlücker, S. (2014) Surface-enhanced Raman spectroscopy: concepts and chemical applications. Angew. Chem. Int. Ed., 53, 4756-4795.

9 Barnes, W.L. (2006) Surface plasmon-polariton length scales: a route to sub-wavelength optics. J. Opt. A: Pure Appl. Opt., 8, S87-S93.

10 Coluccio, M.L., Gentile, F., Das, G., Nicastri, A., Perri, A.M., Candeloro, P., Perozziello, G., Proietti Zaccaria, R., Gongora, J.S.T., Alrasheed, S., Fratalocchi, A., Limongi, T., Cuda, G., and Di Fabrizio, E. (2015) Detection of single amino acid mutation in human breast cancer by disordered plasmonic self-similar chain. Sci. Adv., 1, e1500487.

$11 \mathrm{Yu}$, N. and Capasso, F. (2014) Flat optics with designer metasurfaces. Nat. Mater., 13, 139-150.

12 Atwater, H.A. and Polman, A. (2010) Plasmonics for improved photovoltaic devices. Nat. Mater., 9, 205-213.

13 Mukherjee, S., Libisch, F., Large, N., Neumann, O., Brown, L.V., Cheng, J., Lassiter, J.B., Carter, E.A., Nordlander, P., and Halas, N.J. (2012) Hot electrons 
do the impossible: plasmon-induced dissociation of $\mathrm{H} 2$ on Au. Nano Lett., 13, 240-247.

14 Giugni, A., Torre, B., Toma, A., Francardi, M., Malerba, M., Alabastri, A., Proietti Zaccaria, R., Stockman, M.I., and Di Fabrizio, E. (2013) Hot-electron nanoscopy using adiabatic compression of surface plasmons. Nat. Nanotechnol., 8, 845-852.

15 Kauranen, M. and Zayats, A.V. (2012) Nonlinear plasmonics. Nat. Photonics, 6, 737-748.

16 Huang, J., Liu, C., Zhu, Y., Masala, S., Alarousu, E., Han, Y., and Fratalocchi, A. (2016) Harnessing structural darkness in the visible and infrared wavelengths for a new source of light. Nat. Nanotechnol., 11, 60-66.

17 Lin, J., Genevet, P., Kats, M.A., Antoniou, N., and Capasso, F. (2013) Nanostructured holograms for broadband manipulation of vector beams. Nano Lett., 13, 4269-4274.

18 Park, I.-Y., Kim, S., Choi, J., Lee, D.-H., Kim, Y.-J., Kling, M.F., Stockman, M.I., and Kim, S.-W. (2011) Plasmonic generation of ultrashort extreme-ultraviolet light pulses. Nat. Photonics, 5, 677-681.

19 Rizza, C., Di Falco, A., Scalora, M., and Ciattoni, A. (2015) One-dimensional chirality: strong optical activity in epsilon-near-zero metamaterials. Phys. Rev. Lett., 115, 057401.

20 Govorov, A.O., Zhang, H., Demir, H.V., and Gun'ko, Y.K. (2014) Photogeneration of hot plasmonic electrons with metal nanocrystals: quantum description and potential applications. Nano Today, 9, 85-101.

21 Bernardi, M., Mustafa, J., Neaton, J.B., and Louie, S.G. (2015) Theory and computation of hot carriers generated by surface plasmon polaritons in noble metals. Nat. Commun., 6, 7044.

22 Sundararaman, R., Narang, P., Jermyn, A.S., Goddard Iii, W.A., and Atwater, H.A. (2014) Theoretical predictions for hot-carrier generation from surface plasmon decay. Nat. Commun., 5, 5788.

23 Stockman, M.I. (2004) Nanofocusing of optical energy in tapered plasmonic waveguides. Phys. Rev. Lett., 93, 137404.

24 Stockman, M.I., Kling, M.F., Kleineberg, U., and Krausz, F. (2007) Attosecond nanoplasmonic-field microscope. Nat. Photonics, 1, 539-544.

25 Katrin, K., Harald, K., Irving, I., Ramachandra, R.D., and Michael, S.F. (2002) Surface-enhanced Raman scattering and biophysics. J. Phys. Condens. Matter, 14, R597.

26 Proietti Zaccaria, R., Alabastri, A., De Angelis, F., Das, G., Liberale, C., Toma, A., Giugni, A., Razzari, L., Malerba, M., Sun, H.B., and Di Fabrizio, E. (2012) Fully analytical description of adiabatic compression in dissipative polaritonic structures. Phys. Rev. B, 86, 035410.

27 Ritchie, R.H., Arakawa, E.T., Cowan, J.J., and Hamm, R.N. (1968) Surface-plasmon resonance effect in grating diffraction. Phys. Rev. Lett., 21, 1530-1533.

28 Baron, A., Devaux, E., Rodier, J.-C., Hugonin, J.-P., Rousseau, E., Genet, C., Ebbesen, T.W., and Lalanne, P. (2011) Compact antenna for efficient and unidirectional launching and decoupling of surface plasmons. Nano Lett., 11, 4207-4212. 
29 Kretschmann, E. (1972) The angular dependence and the polarisation of light emitted by surface plasmons on metals due to roughness. Opt. Commun., 5, 331-336.

30 Foley Iv, J.J., Harutyunyan, H., Rosenmann, D., Divan, R., Wiederrecht, G.P., and Gray, S.K. (2015) When are surface plasmon polaritons excited in the Kretschmann-Raether configuration? Sci. Rep., 5, 9929.

31 Otto, A. (1968) Excitation of nonradiative surface plasma waves in silver by the method of frustrated total reflection. Z. Physik, 216, 398-410.

32 De Angelis, F., Das, G., Candeloro, P., Patrini, M., Galli, M., Bek, A., Lazzarino, M., Maksymov, I., Liberale, C., Andreani, L.C., and Di Fabrizio, E. (2010) Nanoscale chemical mapping using three-dimensional adiabatic compression of surface plasmon polaritons. Nat. Nanotechnol., 5, 67-72.

33 Giugni, A., Allione, M., Torre, B., Das, G., Francardi, M., Moretti, M., Malerba, M., Perozziello, G., Candeloro, P., and Fabrizio, E.D. (2014) Adiabatic nanofocusing: spectroscopy, transport and imaging investigation of the nano world. J. Opt., 16, 114003.

34 Lalanne, P., Hugonin, J.P., and Rodier, J.C. (2005) Theory of surface plasmon generation at nanoslit apertures. Phys. Rev. Lett., 95, 263902.

35 Lalanne, P., Hugonin, J.P., and Rodier, J.C. (2006) Approximate model for surface-plasmon generation at slit apertures. J. Opt. Soc. Am. A, 23, $1608-1615$.

36 Proietti Zaccaria, R., De Angelis, F., Toma, A., Razzari, L., Alabastri, A., Das, G., Liberale, C., and Di Fabrizio, E. (2012) Surface plasmon polariton compression through radially and linearly polarized source. Opt. Lett., 37, 545-547.

37 De Angelis, F., Zaccaria, R.P., Francardi, M., Liberale, C., and Di Fabrizio, E. (2011) Multi-scheme approach for efficient surface plasmon polariton generation in metallic conical tips on AFM-based cantilevers. Opt. Express, 19, 22268-22279.

38 Canale, C., Torre, B., Ricci, D., and Braga, P. (2011) in Atomic Force Microscopy in Biomedical Research (eds P.C. Braga and D. Ricci), Humana Press, pp. 31-43.

39 Raether, H. (1988) Surface Plasmons on Smooth and Rough Surfaces and on Gratings, Springer Berlin Heidelberg, Berlin.

40 Burtscher, H., Muller, U., and Schmidt-Qtt, A. (1988) Photoemission from small metal spheres: a model calculation using an enhanced three-step model. Phys. Rev. B, 38, 7814.

41 Scales, C. and Berini, P. (2010) Thin-film Schottky barrier photodetector models. IEEE J..Quantum Electron., 46, 633-643.

42 Petrin, A.B. (2012) Two regimes of nanofocusing of the surface plasmon-polariton wave at the vertex of the metal micropoint. High Temp., 50, 15-20.

43 Knight, M.W., Wang, Y., Urban, A.S., Sobhani, A., Zheng, B.Y., Nordlander, P., and Halas, N.J. (2013) Nano Lett., 13, 1687.

$44 \mathrm{Wu}$, J.C.K., McBride, J.R., and Lian, T. (2015) Efficient hot-electron transfer by a plasmon-induced interfacial charge-transfer transition. Science, 349, 632-635. 
45 Hubenthal, F. (2013) Increased damping of plasmon resonances in gold nanoparticles due to broadening of the band structure. Plasmonics, $\mathbf{8}$, 1341-1349.

46 Govorov, A.O. and Zhang, H. (2015) Kinetic density functional theory for plasmonic nanostructures: breaking of the plasmon peak in the quantum regime and generation of hot electrons. J. Phys. Chem. C, 119, 6181-6194.

47 Esteban, R., Borisov, A.G., Nordlander, P., and Aizpurua, J. (2012) Bridging quantum and classical plasmonics with a quantum-corrected model. Nat. Commun., 3, 825.

48 Akbari, A., Tait, R.N., and Berini, P. (2010) Surface plasmon waveguide Schottky detector. Opt. Express, 18, 8505-8514.

49 Gramotnev, D.K., Vogel, M.W., and Stockman, M.I. (2008) Optimized nonadiabatic nanofocusing of plasmons by tapered metal rods. J. Appl. Phys., 104, 034311.

50 Rangel, T., Kecik, D., Trevisanutto, P.E., Rignanese, G.M., Van Swygenhoven, H., and Olevano, V. (2012) Band structure of gold from many-body perturbation theory. Phys. Rev. B, 86, 125125.

51 Fowler, R.H. (1931) The analysis of photoelectric sensitivity curves for clean metals at various temperatures. Phys. Rev., 38, 45-56.

52 Palik, E.D. (1985) Handbook of Optical Constants of Solids. Part II, Academic Press, New York.

53 Manjavacas, A., Liu, J.G., Kulkarni, V., and Nordlander, P. (2014) Plasmon-induced hot carriers in metallic nanoparticles. ACS Nano, 8, 7630-7638.

54 Zheng, J., Zhang, C., and Dickson, R.M. (2004) Highly fluorescent, water-soluble, size-tunable gold quantum dots. Phys. Rev. Lett., 93, 077402.

55 Rowland, C.E., Fedin, I., Zhang, H., Gray, S.K., Govorov, A.O., Talapin, D.V., and Schaller, R.D. (2015) Picosecond energy transfer and multiexciton transfer outpaces Auger recombination in binary CdSe nanoplatelet solids. Nat. Mater., 14, 484-489.

56 Rohani, S., Quintanilla, M., Tuccio, S., De Angelis, F., Cantelar, E., Govorov, A.O., Razzari, L., and Vetrone, F. (2015) Enhanced luminescence, collective heating, and nanothermometry in an ensemble system composed of lanthanide-doped upconverting nanoparticles and gold nanorods. Adv. Opt. Mater., 3, 1606-1613.

57 Gong, T. and Munday, J.N. (2015) Materials for hot carrier plasmonics [Invited]. Opt. Mater. Express, 5, 2501-2512.

58 Afanas'ev, V.V. (2008) Internal Photoemission Spectroscopy, Elsevier.

59 Donolato, C. (2004) Approximate analytical solution to the space charge problem in nanosized Schottky diodes. J. Appl. Phys., 95, 2184-2186.

60 Smit, G.D.J., Rogge, S., and Klapwijk, T.M. (2002) Scaling of nano-Schottky-diodes. Appl. Phys. Lett., 81, 3852-3854.

61 Sze, S.M. and Ng, K.K. (2007) Physics of Semiconductor Devices, Wiley.

62 Nonnenmacher, M., O’Boyle, M.P., and Wickramasinghe, H.K. (1991) Kelvin probe force microscopy. Appl. Phys. Lett., 58, 2921-2923. 
63 Henning, A.K., Hochwitz, T., Slinkman, J., Never, J., Hoffmann, S., Kaszuba, P., and Daghlian, C. (1995) Two-dimensional surface dopant profiling in silicon using scanning Kelvin probe microscopy. J. Appl. Phys., 77, 1888-1896.

64 Zerweck, U., Loppacher, C., Otto, T., Grafström, S., and Eng, L.M. (2005) Accuracy and resolution limits of Kelvin probe force microscopy. Phys. Rev. B, 71, 125424.

65 Lorenzoni, M., Brandi, F., Dante, S., Giugni, A., and Torre, B. (2013) Simple and effective graphene laser processing for neuron patterning application. Sci. Rep., 3, 1954.

66 Petrin, A.B. (2010) Thermionic-field emission of electrons from conical metal points. High Temp., 48, 305-314.

67 West, P.R., Ishii, S., Naik, G.V., Emani, N.K., Shalaev, V.M., and Boltasseva, A. (2010) Searching for better plasmonic materials. Laser Photonics Rev., 4, 795-808.

68 Berweger, S., Atkin, J.M., Xu, X.G., Olmon, R.L., and Raschke, M.B. (2011) Femtosecond nanofocusing with full optical waveform control. Nano Lett., 11, 4309-4313.

69 Schmidt, S., Piglosiewicz, B., Sadiq, D., Shirdel, J., Lee, J.S., Vasa, P., Park, N., Kim, D.-S., and Lienau, C. (2012) Adiabatic nanofocusing on ultrasmooth single-crystalline gold tapers creates a 10-nm-sized light source with few-cycle time resolution. ACS Nano, 6, 6040-6048.

70 Stockman, M.I., Faleev, S.V., and Bergman, D.J. (2002) Coherent control of femtosecond energy localization in nanosystems. Phys. Rev. Lett., 88, 067402.

71 Mohammadi, A. and Agio, M. (2012) Light scattering under nanofocusing: towards coherent nanoscopies. Opt. Commun., 285, 3383-3389.

72 Bouillard, J.S., Vilain, S., Dickson, W., Wurtz, G.A., and Zayats, A.V. (2012) Broadband and broad angle SPP antennas based on plasmonic crystals with linear chirp. Sci. Rep., 2, 829.

73 Kravtsov, V., Ulbricht, R., Atkin, J.M., and Raschke, M.B. (2015) Plasmonic Nano-Focused Four-Wave Mixing. CLEO: QELS_Fundamental Science, Optical Society of America, 2015, pp. JTh5B. 5.

$74 \mathrm{Xu}$, X.G. and Raschke, M.B. (2013) Near-field infrared vibrational dynamics and tip-enhanced decoherence. Nano Lett., 13, 1588-1595.

75 Min, W., Freudiger, C.W., Lu, S., and Xie, X.S. (2011) Coherent nonlinear optical imaging: beyond fluorescence microscopy. Annu. Rev. Phys. Chem., 62, 507-530.

76 Saar, B.G., Freudiger, C.W., Reichman, J., Stanley, C.M., Holtom, G.R., and Xie, X.S. (2010) Video-rate molecular imaging in vivo with stimulated Raman scattering. Science, 330, 1368-1370.

77 Evans, C.L. and Xie, X.S. (2008) Coherent anti-Stokes Raman scattering microscopy: chemical imaging for biology and medicine. Annu. Rev. Anal. Chem., 1, 883-909.

78 Freudiger, C.W., Min, W., Saar, B.G., Lu, S., Holtom, G.R., He, C., Tsai, J.C., Kang, J.X., and Xie, X.S. (2008) Label-free biomedical imaging with high sensitivity by stimulated Raman scattering microscopy. Science, 322, 1857-1861. 
79 Romera, E. and de los Santos, F. (2009) Revivals, classical periodicity, and zitterbewegung of electron currents in monolayer graphene. Phys. Rev. B, 80, 165416.

80 Rusin, T.M. and Zawadzki, W. (2007) Transient Zitterbewegung of charge carriers in mono- and bilayer graphene, and carbon nanotubes. Phys. Rev. B, 76, 195439.

81 Yakovlev, V., Kruchinin, S., Paasch-Colberg, T., Stockman, M., and Krausz, F. (2016) in Ultrafast Dynamics Driven by Intense Light Pulses (eds M. Kitzler and S. Gräfe), Springer International Publishing, pp. 295-315.

82 Müller, M., Kravtsov, V., Paarmann, A., Raschke, M.B., and Ernstorfer, R. (2016) A nanofocused plasmon-driven sub-10 femtosecond electron point source, ACS Photonics, 3, 611. 